\title{
Epitaxial growth of a colloidal hard-sphere hep crystal and the effects of epitaxial mismatch on crystal structure
}

\author{
J. P. Hoogenboom, ${ }^{1,2, *}$ A. K. van Langen-Suurling, ${ }^{3}$ J. Romijn, ${ }^{3}$ and A. van Blaaderen ${ }^{1,2, \dagger}$ \\ ${ }^{1}$ F.O.M. Institute for Atomic and Molecular Physics, Kruislaan 407, 1098 SJ Amsterdam, The Netherlands \\ ${ }^{2}$ Soft Condensed Matter, Debye Institute, Utrecht University, Princeton Plein 5, 3584 CC Utrecht, The Netherlands \\ ${ }^{3}$ Delft Institute of Microelectronics and Submicron Technology, 2600 GB Delft, Delft, The Netherlands
}

(Received 17 November 2003; published 13 May 2004)

\begin{abstract}
We demonstrate the epitaxial growth of hard-sphere hcp and double hcp crystals using a surface pattern that directly dictates the stacking sequence. A detailed three-dimensional analysis based on real-space measurements is performed on crystal structure as a function of template-crystal mismatch, which demonstrates the possibilities of colloidal epitaxy as a model system for studying the effects of a patterned substrate on crystal structure. Perfect template-induced hcp-crystal growth occurs at an isotropically deformed template. At deformed lattices we observe growth of a non-close-packed superstructure and of a perfect (100)-aligned fcc crystal. Small mismatches lead to increased out-of-plane displacements followed by a structural breakup in "crystal" grains where particle positions in successive layers are strictly periodic and "defect" grains where these positions are displaced with respect to each other. Large mismatches prevent crystallization in the surface layers. The volume fraction was found to vary drastically (up to about 20\%) as a function of template deformation.
\end{abstract}

DOI: 10.1103/PhysRevE.69.051602

PACS number(s): 81.10.Dn, 68.55.-a, 82.70.Dd

\section{INTRODUCTION}

The presence of a periodically modulated external potential, e.g., a substrate, can drastically influence crystallization [1]. In the case of atoms and molecules, epitaxial crystallization has been the subject of intense research over the past decades, mostly driven by its importance for the fabrication of semiconductor devices. For atomic and molecular systems, a systematic evaluation of the interplay between substrate structure and crystallization behavior is however hampered by the difficulties involved in the analysis of structures and in a systematic manipulation of properties on the appropriate length and time scales.

Colloids show phase transitions in thermodynamic equivalence to atomic and molecular systems, but contrary to atoms and molecules their typical length and time scales are easily accessible for experimental techniques such as light microscopy and light scattering [2]. The colloidal model system has already been frequently, and successfully, used for investigating both homogeneous [3-6] crystallization and crystallization close to a wall [7-9]. Recently, these studies have received a great impulse when it was shown that it is possible to perform a full three-dimensional (3D) real-space analysis by using confocal microscopy and fluorescently labeled colloids $[10,11]$. The possibilities of this technique were further demonstrated by several papers addressing hard-sphere glass formation [11-13], crystal nucleation [6],

\footnotetext{
*Author to whom correspondence should be addressed. Present address: Applied Optics Group, MESA ${ }^{+}$Research Institute \& University of Twente, P.O. Box 217, NL-7500AE Enschede, The Netherlands. Email address: j.p.hoogenboom@utwente.nl

${ }^{\dagger}$ Author to whom correspondence should be addressed. Email address: a.vanblaaderen@phys.uu.nl
}

and epitaxial hard-sphere crystallization [14-16]. Several different techniques, e.g., electron-beam lithography, soft lithography [17], and optical tweezers [18], provide the possibility to pattern substrates on length scales comparable to those of colloids [9]. The manipulation of colloidal crystallization by using patterned templates, i.e., colloidal epitaxy $[14,15]$, extends the use of the colloidal model system to include epitaxial crystallization as well. For the simplest colloidal model system, that of Brownian hard spheres, we recently showed how a well-chosen substrate pattern can direct crystallization to an otherwise metastable crystal structure [16].

Apart from this theoretical interest, crystallization of colloidal particles has also gained recent interest from a practical point of view. The fact that the wavelength range of visible light falls in the middle of the colloidal regime not only enables aforementioned experimental techniques, it also makes colloids and colloidal crystals suitable building blocks for photonic applications including photonic band gap materials. The most commonly used techniques for making colloidal crystals for photonic, as well as other applications, rely on self-assembly [19-23]. The ability to direct this selfassembly process in an easy and straightforward way can greatly enhance the possibilities for creating functional colloidal materials. Colloidal epitaxy is in this respect one of the most promising techniques as it gives the possibility to direct crystal structure and orientation [14-16], it is applicable to colloids with a wide range of interaction potentials [24,25], it can be used in combination with other assembly techniques [26,27], and, as we will show in the present paper, provides a way to grow crystals that are metastable in bulk crystallization.

The colloidal model system that we have studied in this research is the conceptually simplest model system that shows a freezing transition upon increasing density, namely, 
the hard-sphere system. This freezing transition is located at a volume fraction of $\varphi=0.494$. For volume fractions in between this volume fraction and the melting transition at $\varphi$ $=0.545$ there is coexistence between a low-density liquid phase and a crystal phase [28,29]. At $\varphi=0.545$ and higher there is a single close-packed crystal phase that compacts at increasing volume fraction. As the interaction potential between hard spheres accounts for excluded volume interactions only, all hard-sphere structures have zero energy. However entropic interactions lead to differences in the total free energy, but these are rather small for the various closepacked structures, on the order of $10^{-4} \mathrm{kT}$ per particle at the melting transition [30,31], where $k$ is Boltzmann's constant.

The equilibrium crystal structure for hard-sphere-like particles is the close-packed face-centered cubic (fcc) crystal, the structure that is highest in free energy is the hexagonal close-packed (hcp) crystal. All the other possible stacking sequences, both regular stackings such as the double hexagonal close packed (dhcp) crystal as well as nonperiodic stacking sequences that are strictly speaking not crystalline have intermediate free energies [30]. Due to the small free-energy differences, stacking faults are likely to occur so that the stacking sequence of hard-sphere crystals can deviate appreciably from the ideal fcc stacking. Furthermore, for small crystallites, with less than $\sim 30000$ particles per plane, a completely random stacking sequence of close-packed planes, denoted with random hexagonal close packed (rhcp), was found to be the most stable structure [31], so crystals grown via a nucleation-and-growth-mechanism will initially have a rhcp structure. This is in correspondence with observations in space [32] and on earth [3,33-35]. The subsequent relaxation of the rhcp structure to fcc is estimated to be slow, with time scales on the order of months to years for $200 \mathrm{~nm}$ diameter particles [31,36,37].

One of the techniques that was shown to lift this apparent degeneracy and induce formation of a perfect fcc stacking is colloidal epitaxy. Furthermore, it was shown possible to orient the crystal with both the (100)-plane [14] as well as the (110)-plane [15], which has an even higher surface free energy, aligned over the bottom, templated wall. In this paper, we will show that it is not only possible to direct hard-sphere (HS) crystallization towards the crystal structure with the lowest free energy, but that the most metastable crystal structure in bulk, the hcp stacking, can be grown as well. The template that will be used for hcp-crystal formation, the hcp(1100) plane, can be easily extended to direct any of the other possible stacking sequences, which will be shown by growing a so-called double hcp (dhcp) crystal. These results are interesting in view of recent theoretical work on HS crystallization on structured walls that predicted complete wetting of the metastable hcp crystal at a suitably patterned substrate $[38,39]$. Other theoretical predictions, such as the fact that the optimal lattice constants of the template are larger than those of the bulk crystal, are reproduced in this research as well. Furthermore, Pronk and Frenkel have recently calculated that the application of moderate deformations of the hard-sphere crystal structure may change the relative stability of fcc and hep crystals to the order of $10^{-2} k_{\mathrm{B}} T$ per particle in favor of hcp [40].

Isotropically and anisotropically stretched hcp(1100)template lattices were used to study the effects of template- crystal mismatches on epitaxial crystallization. Among our observations is the growth of a superstructure that consists of six differently stacked non-close-packed planes with a stretched hcp(1100) symmetry. The occurrence of a nonclose-packed HS crystal has, to our knowledge, never been predicted theoretically. The possibility to create metastable and non-close-packed crystal structures opens up ways to unexplored photonic crystals.

Two other results clearly show the potential of colloidal epitaxy together with three-dimensional real-space analysis as a model system for epitaxial crystallization and as a tool to study defect formation and (epitaxial) stress relaxation in molecular crystals. At a template unit cell of which both lattice vectors have the same relative amount of shrinkage with respect to the unit cell that gives rise to hcp crystallization, we observe a reconstruction of the colloidal surface layer towards an fcc(100) plane, similar to (100)-hcp(1100) reconstructions observed in atomic heteroepitaxy of, e.g., Co and $\mathrm{Cu}$ [41-43]. Second, a mismatch between template and crystal dimensions is in general found to lead to an increase in density in between lattice planes [41,42]. A thorough understanding of and control over defect formation is furthermore crucial for photonic applications of colloidal materials [44]. In this respect, the ability to exactly control the stacking sequence of a colloidal crystal allows for a direct experimental test of theoretical work on the effect of stacking faults on the optical properties of inverse opals [45].

The remainder of this paper is organized as follows. First, we will discuss our experimental model system and the various order parameters that we calculate in order to examine colloidal crystal structure. Then we will present and discuss results on the growth of hcp and dhcp crystals, after which we will focus on the evolution of crystal structure as a function of template-crystal mismatch. The starting point for this will be the investigation of unit cells that are isotropically shrunken with respect to the hcp(1100) template that gives rise to the best quality hcp crystal. This analysis includes the epitaxial hcp(1100)-fcc(100) transition. Then results for an isotropically stretched template will be presented, followed by an anisotropically stretched template, where the ratio between the two lattice distances is increased. Finally, the occurrence of a non-close-packed superstructure will be discussed.

\section{EXPERIMENTAL DETAILS}

\section{Core-shell colloids}

Silica colloids with core-shell morphology were used in order to make quantitative three-dimensional (3D) confocal microscopy possible. The core of the particles contained the fluorescent dye fluorescein isothiocyanate (FITC) covalently attached to the silica through the silane coupling agent 3-aminopropyltriethoxysilane. The synthesis of these particles has been described in detail [46]. The core diameter was measured with both static light scattering (SLS), $d$ $=400 \mathrm{~nm}$, and transmission electron microscopy (TEM), $d$ $=386 \mathrm{~nm}$. These core particles were grown larger with an unlabeled silica shell using a seeded growth procedure as described by Giesche [47]. For the final particle diameter we 
found $d=1.384 \mu \mathrm{m}$ by TEM and $d=1.404 \mu \mathrm{m}$ by SLS. The polydispersity, defined as the standard deviation of particle sizes relative to the mean, was determined by TEM to be 0.015. In the seeded growth procedure that was used to cover the fluorescently labeled core particles with a nonfluorescent shell, instabilities in the reaction mixture may affect particle growth in two unfavorable ways. First, an uncontrolled, local increase in counterion concentration may lead to the formation of aggregated doublet particles, so-called dumbbells. Second, an uncontrolled increase in the local concentration of silicic acid may lead to the nucleation of smaller, nonfluorescent particles, instead of condensation on the surface of the seed particles. In the final dispersion that was used the concentration of both "impurities" was below $1 \%$ of the particle concentration.

Particles were suspended in a refractive-index matching solution of demineralized water and glycerol (Sigma) in a volume ratio of $1: 6.5$. The conditions for refractive-index matching were determined by measuring the transmission of different mixtures two days after dispersing a low volume fraction of particles. Due to the refractive-index matching the van der Waals attractions between the particles are reduced, leading, for particles in this size range, to a steeply repulsive interaction due to surface charges and residual counterion concentrations. This results in an almost hard-sphere-like interaction. In order to calculate an effective hard-sphere radius $R_{H S}$, we calculated the 3D radial distribution function, 3D $g(r)$, defined as

$$
g(r)=\frac{1}{\rho^{2}}\left\langle\delta\left(r-r_{i}\right) \delta\left(r-\left(r_{i}-r_{j}\right)\right)\right\rangle
$$

for a crystal grown in the same sample as that for the experiments described below, but at a plain, untemplated wall. From the position of the first maximum we found $2 R_{H S}$ $=1.460 \mu \mathrm{m}$. For a close-packed crystal this corresponds to a volume fraction of $\varphi=0.65$.

\section{The sample cell and the hcp(1100) template}

A sample cell was made by gluing (General Electric Silicon Rubber Adhesive RTV 102) a bottomless bottle with an internal diameter of $12.85 \mathrm{~mm}$ to a $22 \mathrm{~mm}$ diameter glass slide (chance no. 1) that had been coated and partly patterned as described below. The sample cell was rinsed several times with the water-glycerol mixture before filling it with about $1.2 \mathrm{ml}$ of colloidal suspension. Colloids were homogeneously dispersed with a volume fraction of $\varphi_{0}=0.0015$. For the experiments with anisotropically deformed template unit cells (see the discussion on the template patterns below) suspensions with an initial volume fraction of $\varphi_{0}=0.005$ were used. By sedimentation, colloids accumulated and crystallized at the bottom wall.

With the initial volume fractions and total volume given above, the volume fraction at the bottom of the sample, $\varphi_{1}$, can be calculated, equating the gravitational pressure to the balancing osmotic pressure:

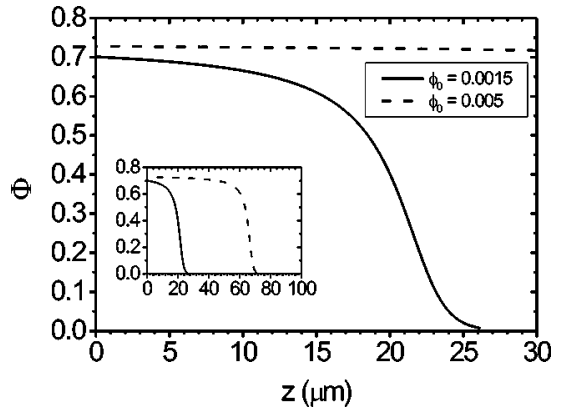

FIG. 1. Calculated dependence of the volume fraction in the sediment as a function of height for hard spheres at the two different initial volume fractions used in this study. The straight curve with $\varphi_{0}=0.0015$ corresponds to the experiments with the isotropically stretched and shrunken templates; the dashed curve with $\varphi_{0}$ $=0.005$ corresponds to the experiments with the anisotropically stretched templates. In the inset the decay of the $\left(\varphi_{0}=0.0015\right)$ graph is visible as well.

$$
\varphi_{1}=\frac{\varphi_{0} \mathrm{Pe}(H / R) \varphi_{m}}{3 \varphi_{m}+\varphi_{0} \mathrm{Pe}(H / R)}
$$

where $H$ is the total height of the sample solution from the bottom wall to the meniscus, $R$ is the particle radius, $\varphi_{m}$ is the volume fraction at maximum compression, which is $\varphi_{m}$ $=0.74$ for crystallized hard spheres. Pe is the Peclet number which is defined as $\mathrm{Pe}=4 / 3 \pi R^{4}(\Delta \rho) g /(k T)$ with $\Delta \rho$ the density difference between particles and solvent, $g$ the gravitational constant, $k$ Boltzmann's constant, and $T$ the temperature. For our initial volume fractions of $\varphi_{0}$ $=0.0015$ and $\varphi_{0}=0.005$, we get values for $\varphi_{1}$ of $\varphi_{1}=0.70$ and $\varphi_{1}=0.73$, respectively. With these values, the volume fraction profile of the sediment is given according to [48]

$$
\operatorname{Pe} \frac{h}{R}=\left[\frac{3 \varphi_{m}}{\varphi_{m}-\varphi}+3 \ln \left(\frac{\varphi}{\varphi_{m}-\varphi}\right)\right]_{\varphi(h)}^{\varphi_{1}},
$$

where $h$ is the height from the bottom wall. The calculated volume fraction profiles for hard spheres are given in Fig. 1.

The bottom wall consisted of a glass slide (chance no.1), coated successively with an $11.5 \mathrm{~nm}$ thick gold layer and a $450 \mathrm{~nm}$ thick poly(methylmethacrylate) (PMMA) layer. The PMMA (MicroChem Corp., $950 \mathrm{k}, 4 \mathrm{wt} \%$ in chlorobenzene) layer was doped with the fluorescent dye pyromethene 580 (Exciton Inc.) by dissolving typically $10^{-3}$ w.t $\%$ of dye in the PMMA solution prior to spin coating. This PMMA layer was partly patterned using electron-beam lithography. Each single pattern consisted of $500 \times 500$ unit cells. The depth of the circular holes in the pattern was $450 \mathrm{~nm}$, equivalent to the PMMA-layer thickness.

On a plain, flat, hard wall hard-sphere particles crystallize in close-packed hexagonal layers parallel to this surface. These hexagonal layers correspond to the (111) plane of the fcc crystal. In Fig. 2 a sketch of particle positions in this layer is given. For the next hexagonal layer there are two distinct stacking possibilities, denoted by $B$ and $C$, while positions in the first layer are indicated by $A$. The template we have chosen for our experiments is a crystal plane that 


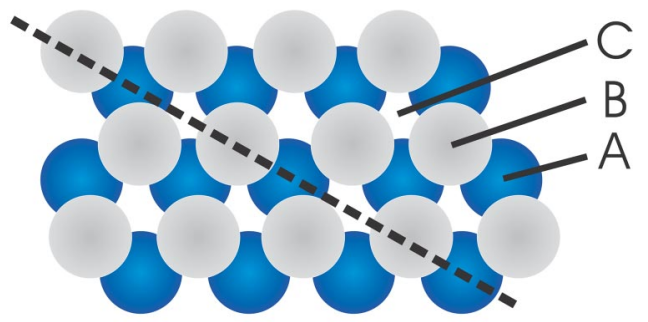

FIG. 2. (Color online) Schematic drawing of close-packed hexagonal planes in a hcp crystal, with the $A, B$, and $C$ positions indicated. The hcp(1100) plane is perpendicular to the close-packed planes, its orientation indicated by the dashed line.

runs perpendicular to the hexagonal ground plane and contains the $A, B$, and $C$ positions, as indicated by the dashed line in Fig. 2. For fcc and hcp crystals this corresponds to the fcc(110) plane and hcp(1100) plane, respectively, where for the fcc lattice crystal planes are denoted with the indices of the three cubic lattice vectors, while for the hcp lattice we use the three vectors associated with the hexagonal ground plane and the direction perpendicular to this as the fourth index. In order to only distinguish fcc and hep stackings there are more possible choices for the template plane, for instance, fcc(110) vs hcp (1100) [38,39], but the hcp(1100) plane gives us the further advantage to directly dictate any of the intermediate stacking sequences as well. A confocal image of the resulting hcp(1100) template is given in Fig. 3(a). An example of another stacking sequence dictated by the template, namely, the $A B C B A$ sequence, is given in Fig. 6(a). This sequence corresponds to a dhcp crystal.

The dimensions of the hcp(1100) unit cell are given by the distances $a$ and $c$, indicated in Fig. 3(a). For spheres of radius $R$, these distances are given by $a=2 R \sqrt{ } 3$ and $c$ $=4 R \sqrt{ }(2 / 3)$. The length of the $c$ vector is twice the distance between close-packed planes. The coordinates of the $B$ position in the unit cell are given by $(a / 3, c / 2)$. The actual values of $a$ and $c$ were varied by stretching or shrinking the unit cell along these two directions. The resulting dimensions were scaled to the effective hard-sphere dimensions $a_{H S}$ $=2 R_{H S} \sqrt{ } 3$ and $c_{H S}=4 R_{H S} \downarrow(2 / 3)$, with $R_{H S}$ calculated as described above. The values of $a / a_{H S}\left(=c / c_{H S}\right)$ are given in

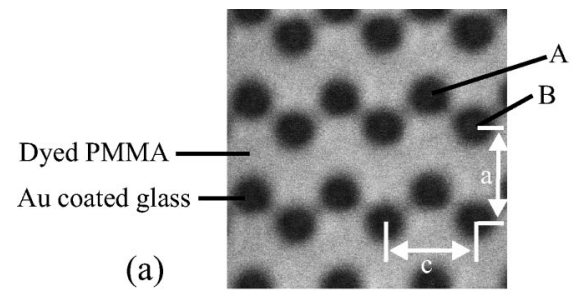

(b)

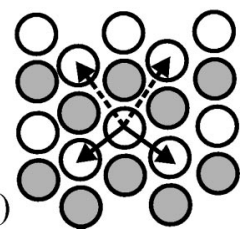

FIG. 3. (a) Confocal microscopy image of a hcp(1100) template, with the characteristic $A B A$ sequence of the close-packed planes that grow perpendicular to the template indicated. The distances a and $\mathrm{c}$ span the 2D unit cell and their lengths are in this image 2.08 and $1.96 \mu \mathrm{m}$, respectively. (b) Drawing of the projection of sphere positions in two successive hcp(1100) layers. The solid arrows indicate the in-plane nearest-neighbor distance; the dashed arrows the next-nearest-neighbor distance.
Table I. In the remainder of this paper we will denote deformations with $a / a_{H S}=c / c_{H S}>1$ as positive, isotropic deformations, deformations with $a / a_{H S}=c / c_{H S}<1$ as negative, isotropic deformations, and finally deformations with $a / a_{H S}$ $\neq c / c_{H S}$, where always $c / c_{H S}>a / a_{H S}$, as anisotropic deformations.

\section{Structural analysis}

After sedimentation, samples were analyzed with an inverted confocal microscope (Leica TCS-NT 2). The $488 \mathrm{~nm}$ line of an ArKr laser was used to excite the FITC fluorophores in the particle cores. The use of particles with a nonfluorescent shell as described above allows for a 3D analysis. 2D images parallel to the optical axis were taken with a typical spacing along the optical axis (further denoted as the $z$ direction) of $\Delta z=122 \mathrm{~nm}$. In each of these images feature coordinates and intensities were retrieved using procedures similar to those described by Crocker and Grier [49]. Features belonging to a single-sphere's intensity profile were then grouped together in columns sorted on their $z$ coordinate. This intensity profile is the $z$ component of a convolution of the point spread function (PSF) of our microscope with the spherical profile of the core particles. A Gaussian function was constructed of which the full width at half maximum was fixed at a value that was such that this Gaussian function mimicked the $z$ axis component of the convolution of the PSF with the spherical core profile. Each of the intensity profiles in the 3D data set was then fitted to this Gaussian and the position of the maximum was taken as a particle's $z$ coordinate. This $z$ coordinate has an accuracy of $\pm 30 \mathrm{~nm}$.

With the sets of 3D particle coordinates that were thus obtained, an analysis of crystal structure was carried out. Our analysis started by calculating the linear number density perpendicular to the bottom wall by integration of the 3D density profile over the lateral $(x y)$ coordinates

$$
\rho_{z}=\frac{\int_{z-\Delta z / 2}^{z+\Delta z / 2} \iint \delta\left(x-x_{0}\right) \delta\left(y-y_{0}\right) \delta\left(z-z_{0}\right) d x d y d z}{\Delta z \int d x d y},
$$

where the bin size $\Delta z=0.040 \mu \mathrm{m}$.

At a plain wall, hard spheres crystallize in hexagonal layers oriented parallel to the bottom wall. For a templated crystal we also expect crystal planes to be oriented parallel to the (templated) bottom wall, but with an in-plane symmetry dictated by the template. In both cases the positions of these layers will be visible by a peaked structure of $\rho_{z}$. Based on the minima in $\rho_{z}$ particles were assigned to layers parallel to the bottom wall.

Volume fractions were calculated by direct particle counting in layers 3-10 from the bottom. These volume fractions were scaled to the hard-sphere volume fraction. As can be seen in Fig. 1, the volume fraction in this range of heights may, for the sample with $\varphi_{0}=0.0015$, change depending on the layer spacing. In order to account for the decreasing vol- 
TABLE I. Values for the various order parameters for isotropic template deformations.

\begin{tabular}{lcccccccc}
\hline \hline$a / a_{H S}$ & $\left\langle\psi_{6}^{\text {poj }}\right\rangle_{3,4}$ & $\langle\xi\rangle_{3}$ & $\left\langle\psi_{4}\right\rangle_{3}$ & $\left\langle\psi_{6}^{\text {proj }}\right\rangle_{10,11}$ & $\langle\xi\rangle_{10}$ & $\left\langle\psi_{4}\right\rangle_{10}$ & Structure $^{\mathrm{a}}$ & $\varphi / \varphi_{H S}^{\mathrm{b}}$ \\
\hline 0.753 & 0.35 & 0.09 & 0.41 & 0.36 & 0.18 & 0.44 & DIS & 0.84 \\
0.822 & 0.24 & 0.08 & 0.62 & 0.29 & 0.16 & 0.52 & fcc & 0.74 \\
0.890 & 0.21 & 0.11 & 0.93 & 0.25 & 0.22 & 0.72 & fcc & 0.77 \\
0.959 & 0.34 & 0.23 & 0.86 & 0.46 & 0.19 & 0.80 & fcc & 0.90 \\
0.986 & 0.58 & 0.66 & 0.84 & 0.34 & 0.43 & 0.85 & H-F & 0.85 \\
1.055 & 0.73 & 0.84 & 0.72 & 0.72 & 0.83 & 0.73 & hcp & 0.92 \\
1.068 & 0.73 & 0.82 & 0.71 & 0.73 & 0.83 & 0.72 & hcp & 0.82 \\
1.151 & 0.66 & 0.98 & 0.57 & 0.75 & 0.80 & 0.66 & hcp & 0.88 \\
1.247 & 0.33 & 0.81 & 0.44 & 0.32 & 0.86 & 0.45 & DIS & 0.81 \\
\hline \hline
\end{tabular}

${ }^{\mathrm{a}}$ DIS stands for disordered, H-F for hep in layer 3 to fcc in layer 10.

${ }^{\mathrm{b}}$ The error margin in the volume fraction is $\pm 5 \times 10^{-3}$.

ume fraction in the sample, the hard-sphere volume fraction was determined by averaging the volume fraction as given in Fig. 1 over the same range in $z$.

For the particles in each layer we calculated three order parameters that together describe the effect of the (deformed) template on crystal structure. In the following we will indicate layers with the index $i$ and the 2D particle coordinates in layer $i$ with $r_{i}=(x, y)$. First, we calculated the in-plane 2D analog of the radial distribution function. The definition is equal to that given in Eq. (1), with the exception that now the integration only runs over the lateral coordinates of particles whose $z$ coordinate falls between the two minima in $\rho_{z}$ that define the layer. We denote this function with $2 \mathrm{D} g(r)$ to indicate the difference with the 3D $g(r)$ defined above. Second, a measure for the asymmetry of the distribution of nearest-neighbor and next-nearest-neighbor distances was calculated. As can be seen in Fig. 3(b), the first four nearest neighbors in the hcp(1100) unit cell occupy two sets of distinct distances, $\left\{\vec{r}_{1}, \vec{r}_{2}\right\}$ (indicated with the solid arrows) vs $\left\{\vec{r}_{3}, \vec{r}_{4}\right\}$ (indicated with the dashed arrows), where the order $(1,2,3,4)$ is determined by increasing distance from the center particle. For a hexagonal, fcc(111) plane, as well as, for instance, for a fcc(100) plane, all these four distances have similar values. In order to quantify this distribution we have used the function $\xi$ that, for a particle in layer $i$ at position $r_{j}$, is defined as

$$
\xi\left(r_{j}\right)=\frac{1}{\sqrt{2}-1}\left(\frac{\left|\vec{r}_{j 3}\right|}{\left|\vec{r}_{j 2}\right|}-1\right)
$$

where $r_{j 2}$ and $r_{j 3}$ denote the position of the second and third neighbors of particle $j$ with the order as given above. The scaling is used to map the value of $\xi$ for an ideal hcp(1100) lattice to 1 . Note however that the possible values for this parameter are not limited to $\langle 0,1\rangle$. The value for $\xi$ can continuously vary and may become larger than 1 . It only gives a measure of the asymmetry of nearest-neighbor distances in comparison to that in the hcp(1100) lattice. However, a pure fcc(110) lattice (that we however did not find to occur in our experiments, except for local stacking faults at high template deformations) will give identical values as the hcp(1100) lattice.

The $\xi$ parameter was found to give identical results as using the mean-squared-distance (msd) between neighboring particles, summed over each particle's four nearest neighbors. However, the distributions of values for $\xi$ for hcp(1100) and the symmetric unit cells listed above were found to be more clearly separated than for the msd criterion. Furthermore, we only included the distances to second and third nearest neighbors, as this was found to give better results than using all first four nearest-neighbor distances, probably due to the influence of defects. For instance, with the definition given above, a vacancy in a hcp(1100) lattice will map the result for half of its neighbors to zero and for the other half to one. Thus it will not give any intermediate values.

In order to also quantify orientational order in each layer, the $2 \mathrm{D}$ local hexagonal bond-orientational order parameter [50] was calculated for a projection of particle positions $r_{i, i+1}$ from two successive layers:

$$
\psi_{6}^{p r o j}\left(r_{j}\right)=\frac{1}{N_{b}} \sum_{k} \exp \left[i 6 \theta\left(r_{j k}\right)\right]
$$

where the summation $k$ runs over all, in total $N_{b}$, neighboring particles of a particle $j$ in layers $i$ and $i+1$, and the angle $\theta$ is the angle that the bond vector between particles $j$ and $k$ makes with an arbitrary fixed reference axis. As can be seen in Fig. 3(b), a projection of hcp(1100) layers gives an almost hexagonal symmetry and thus a distribution of values for $\psi_{6}^{\text {proj }}$ close to 1 . For other possible hard-sphere crystal planes such as fcc(111) and fcc(100), a two-layer projection does not give rise to projected hexagonal symmetry. With the parameters $\xi$ and $\psi_{6}^{p r o j}$ a global classification of (hcp) crystal structure is given. If the layer-averaged (or in the case of $\psi_{6}^{p r o j}$ two-layer averaged) values $\langle\xi\rangle_{i}$ and $\left\langle\psi_{6}^{\text {proj }}\right\rangle_{i, i+1}$ both give a value larger than 0.5 , crystal structure is denoted as hep.

In order to discriminate the fcc(100) symmetry the singlelayer fourfold bond-orientational order parameter, defined as in Eq. (6), but with a factor 4 instead of 6 in the exponent and with a scaling of the value of $\psi_{4}$ with the nearestneighbor distance, was calculated as well. If the parameters $\xi$ 
and $\psi_{6}^{\text {proj }}$ gave average values smaller than 0.5 while the average $\left\langle\psi_{4}\right\rangle_{i}$ was larger than 0.5 , the structure was labeled as fcc(100). Furthermore, a hexagonal lattice was identified with the single-layer averaged sixfold bond-orientational order parameter being larger than 0.5. Finally, a structure was denominated as disordered when the density profile did not show clear, periodic minima except for a few layers over the template (we always found layering to occur at the bottom wall) and the order parameters defined before did not correspond to any of the criteria given for these first four layers, which typically meant that $\langle\xi\rangle_{i}$ was larger than 0.5 , while $\left\langle\psi_{6}^{p r o j}\right\rangle_{i, i+1}$ was smaller than 0.5 .

Apart from the order parameters defined above that were used to identify the structure over the template, we have also examined the extent to which particle coordinates in successive layers followed the positions dictated by the template. For this the coordinates that correspond to the positions given by the template and the second layer of the ideal 3D crystal of which the template layer is the first were used. These coordinates were denoted with $\vec{r}_{T}$. Starting from these positions, particles differing in height in the sample were connected by linking positions $\vec{r}_{T}$ to the nearest particle coordinates in the first two layers, with a cutoff radius of $0.5 \mu \mathrm{m}$, and repeating this procedure with these coordinates and that in the next two layers. Such a set of connected $x y$ positions of particles in the sample will be denoted as the lateral displacement curve (LDC). For all LDC's that were retrieved in this way, the $2 \mathrm{D}$ root-mean-squared displacement relative to the template was calculated as

$$
\Delta_{n}=\left\langle\left|\vec{r}_{n}-\vec{r}_{T}\right|^{2}\right\rangle,
$$

where $n=2 i+1$ and $i$ is the layer index starting from the template $\left(\left\{\vec{r}_{1}, \vec{r}_{2}\right\}=\vec{r}_{T}\right)$. In order not to be affected by point defects such as vacancies, an intermediate lattice position is allowed to be unoccupied, with the LDC being "restored" afterwards. For a perfect epitaxially grown crystal, the lattice positions $\vec{r}_{T}$ are periodically repeated in all crystal planes parallel to the template. Then the root-mean-squared displacements $\Delta n$ are just the vibrations of a particle around its lattice site and the distribution of $\Delta n$ for all $n$ will have a maximum at a relatively small, nonzero value. For a sample where there is hardly any correlation between successive layers and thus between these layers and the template, LDC's will still be constructed, though now the distribution will flatten between zero and the cutoff radius, and furthermore due to the small difference between cutoff radius and particle radius, resp. $0.5 \mu \mathrm{m}$ and $0.77 \mu \mathrm{m}$, and the fact that a particle is allowed to disappear in one intermediate layer, there will be a peak for small values of $\Delta$ corresponding to restored, uncorrelated particle positions. This last fact can also be observed when examining the extent to which LDC's persist through the sample. The plots of all 2D LDC's will provide information about correlations in the direction of particle displacements in successive layers.

\section{RESULTS}

\section{hep and dhep crystals}

In Fig. 4 confocal images of a crystal grown on a template with lattice constants of $a=2.67 \mu \mathrm{m}$ and $c=2.51 \mu \mathrm{m}$ are

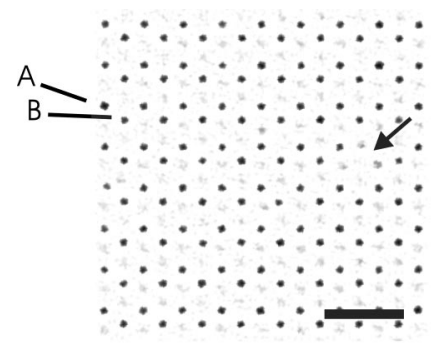

(a)

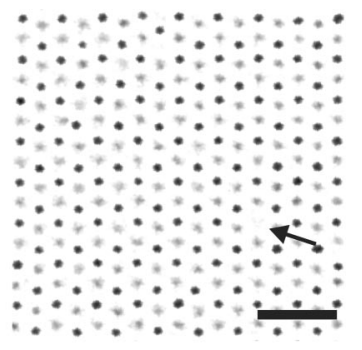

(b)
FIG. 4. Confocal images of (a) the first layer of a templated hcp crystal and (b) the 18th layer in the crystal, with the 19th layer vaguely visible as well. The arrows indicate the positions of vacancies in the crystal. Scale bars are $5 \mu \mathrm{m}$.

shown. Figure 4(a) shows the first crystal layer over the template, and as can be seen the template symmetry [Fig. 3(a)] is fully replicated by the silica particles. In Fig. 4(b), denoting the 18th layer in the crystal, corresponding to a depth of $\approx 14 \mu \mathrm{m}$, it can be seen that the $A B A$ stacking is still preserved, showing the growth of a perfect, stacking-fault-free hcp crystal. The hcp crystal was found to be monocrystalline over the full area of the template $\left(1.33 \times 1.26 \mathrm{~mm}^{2}\right)$. Furthermore, hcp crystal structure was found to extend as far as the thickness of our crystal, which was $\approx 20 \mu \mathrm{m}$.

As mentioned in the preceding section, we give a classification of (hcp) crystal structure as a function of templatecrystal mismatch, based on the values for $\langle\xi\rangle_{i}$ and $\left\langle\psi_{6}^{p r o j}\right\rangle_{i, i+1}$. This however is just an indicative qualification, with, as we will see in detail below, the occurrence of defects and especially dislocations slowly increasing at increasing absolute deformations. For negative isotropic deformations with $a / a_{H S}$ from 0.959 to 0.822 , we observe a reconstruction to a fcc crystal structure with the (100) plane aligned on the template. For a deformation of $a / a_{H S}=0.986$, order parameters for the bottom layers indicate a hcp structure, while for the tenth layer from the bottom the in-plane structure is of fcc(100)-type. For large deformations, $a / a_{H S}=0.753$ and $a / a_{H S}=1.397$, strong distortions of the hcp crystal lead to disorder in the first few layers above the template after which a hexagonally stacked crystal that is tilted with respect to the bottom wall develops. The values for all order parameters for the isotropic deformations are given in Table I. Note that for the hcp (1100)-oriented crystals, $\left\langle\psi_{4}\right\rangle_{i}$ has a value higher than 0.5 as well, though these values are smaller than those for the fcc crystals. The resulting evolution of crystal structure as a function of stress caused by an isotropic stretch relative to the effective hard-sphere unit cell is given in Fig. 5. Results for anisotropic stretches of the template, where the axis ratio $\left(c / c_{H S}\right)\left(a / a_{H S}\right)^{-1}$ was varied, are indicated in Fig. 5 as well.

As visible in Fig. 5, the stability regime of the hcp crystal is remarkably shifted from the values one would expect based on the effective hard-sphere diameter that was calculated in the same sample on an untemplated part of the bottom wall. As we will see below, a perfectly layered hcp crystal was found at $a / a_{H S}=c / c_{H S}=1.055$.

As mentioned in the preceding section, the stacking sequence in the $h c p(1100)$ plane is directly dictated by the template. As the hcp crystal structure is the close-packed 


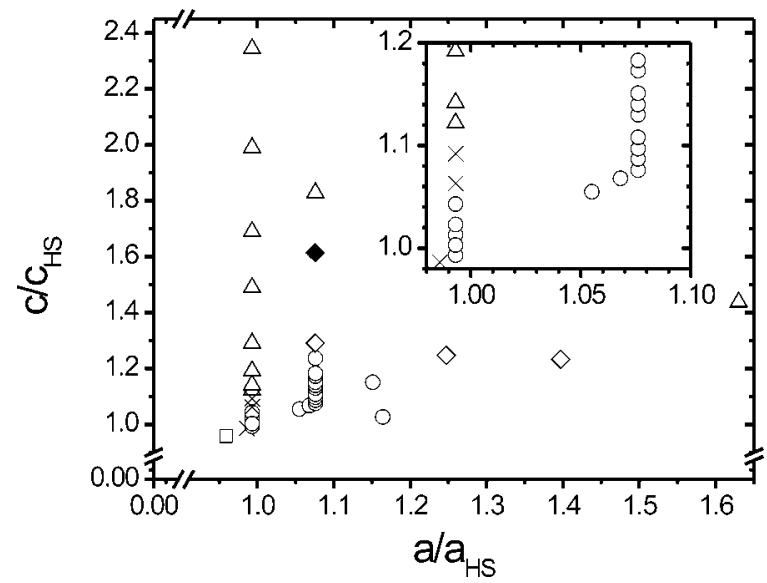

FIG. 5. Schematic graph of crystal phases vs scaled lattice constants. The open symbols denote the occurrence of a stable phase from the template onwards: hcp $(\bigcirc)$, fcc $(\square)$, or hexagonally stacked $(\Delta)$; crosses $(\mathrm{x})$ indicate that the structure was hcp $(1100)$ in the third layer, while fcc(100) in the tenth layer. The filled diamond indicates a non-close-packed structure. The open diamonds denote a disordered state in the first layers above the template.

hard-sphere stacking sequence with the highest free energy [30] the above result indicates that it should be possible to incorporate stacking faults in any desired position in a fcc or hcp crystal by simply shifting one row of positions in the template over a distance of $\pm a / 3$. In this way one could, for instance, experimentally test theories on the influence of stacking faults on the optical properties of photonic crystals [45]. We have checked this possibility by using an $A B C B A$ template, which corresponds to the stacking of a dhcp crystal. Figure 6(a) shows the dhcp template with the $A B C B A$ sequence visible. In this image colloids in the first, perfectly templated, crystal layer are visible as well within the template. In Fig. 6(b) the tenth layer is shown. The total thickness of the crystalline part of the sediment of about $20 \mu \mathrm{m}$ corresponds to a perfectly stacked dhcp crystal of 30 layers. For the dhcp crystal we observed a similar shift of the stability regime to positive deformations of the unit cell as for the hcp crystal.

Furthermore, perfectly layered dhcp crystal growth was found to occur, within the experimental resolution determined by the discrete values of $a / a_{H S}$, at the same $a / a_{H S}$ as for the hcp crystal that we will analyze below. At negative

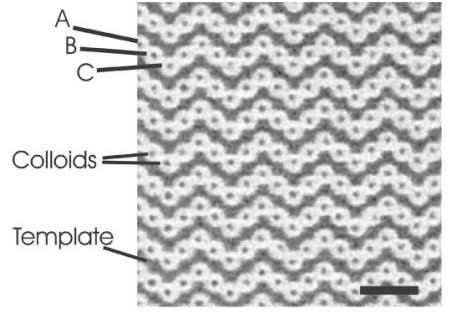

(a)

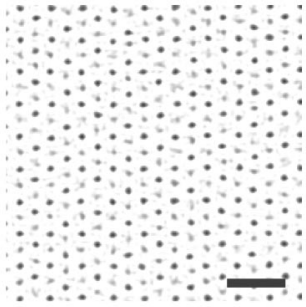

(b)
FIG. 6. Confocal images of (a) template and particles in the first layer of a dhcp $(A B C B A)$ crystal and (b) the tenth layer at a depth of $8 \mu \mathrm{m}$. The length of the scale bar is in both images $5 \mu \mathrm{m}$. deformations of the unit cell, also the dhcp template gives rise to a surface reconstruction towards fcc(100), although we did not, at the deformations studied, observe a perfectly layered, defect-free fcc crystal as in the case of the hcp crystal.

\section{Negative isotropic deformations and the fcc crystal}

In Fig. 5 and Table $I$ it can be seen that an isotropic shrinkage of the hcp(1100) unit cell leads to fcc crystallization. We will first look at the evolution of crystal structure as a function of unit cell deformation for this hep-fcc transition starting from the hcp-stability regime. In Fig. 7 the first ten peaks (layers) in the linear number density $\rho_{z}$ are given for various values of $a / a_{H S}$ ranging from 1.055 to 0.753 . Here, the position of the first peak in $\rho_{z}$ has been taken as $z=0$, as we did not retrieve the exact position of the bottom wall from our confocal data sets. As can be seen, we start in Fig. 7(a) with a perfectly layered structure, where the density in between layers falls to zero. This is expected for a templated crystal where crystal planes run parallel to the patterned bottom wall. The crystal shown in Fig. 7(a) corresponds to the confocal images shown in Fig. 4, i.e., the hcp crystal. From the linear number density in Fig. 7(a) follows an interlayer spacing of $\Delta z=0.73 \pm 0.01 \mu \mathrm{m}$ in correspondence with the value of $\Delta z_{H S}=R_{H S}=0.730 \mu \mathrm{m}$ expected for a hcp(1100) plane based on the effective hard-sphere radius and an osmotic pressure that gives rise to almost close-packed conditions.

At smaller $a / a_{H S}$, the width of the peaks increases, which is accompanied by a decreasing peak height, an increasing interlayer density, and an increase in the total height of the first ten layers [note the changing scale on the $z$ axis in Figs. 7(a)-7(f)]. These are all indications of out-of-plane displacements of particles, and, at further decreasing $a / a_{H S}$, small grains of particles that move up or down with respect to the average layer position. In between $a / a_{H S}=0.986$ and $a / a_{H S}$ $=0.959$ this leads to overlapping of successive peaks, and, at $a / a_{H S}=0.959$, a uniform density with oscillations after the first two layers.

This trend is however interrupted at $a / a_{H S}=0.890$ [Fig. 7(d)], where crystal structure is again perfectly layered, with interlayer density sharply falling to zero. This situation corresponds to a (100)-oriented fcc crystal structure. Note that in Fig. 7(d) also the scale on the $\rho_{z}$ axis has changed with respect to the other graphs in Fig. 7. This, together with the increased interlayer distance is in correspondence with the higher in-plane density of the fcc(100) plane compared to the hcp(1100) plane. For the interlayer spacing in Fig. 7(d) we find $\Delta z=1.14 \pm 0.01 \mu \mathrm{m}$, which is a factor of 1.11 higher than the value of $\Delta z_{H S}=1.03 \mu \mathrm{m}$ that would be expected based on the effective hard-sphere radius. Even when we would take an interparticle distance that corresponds to the stretched hcp(1100) lattice $(d=1.54 \mu \mathrm{m})$, the interlayer spacing of $\Delta z=1.14 \pm 0.01 \mu \mathrm{m}$ for the (100)-oriented fcc crystal is still a factor 1.05 higher than the value of $\frac{1}{2} d \sqrt{ } 2$ that would be expected.

At larger negative deformations of the template unit cell than $a / a_{H S}=0.890$, the same behavior of out-of-plane dis- 

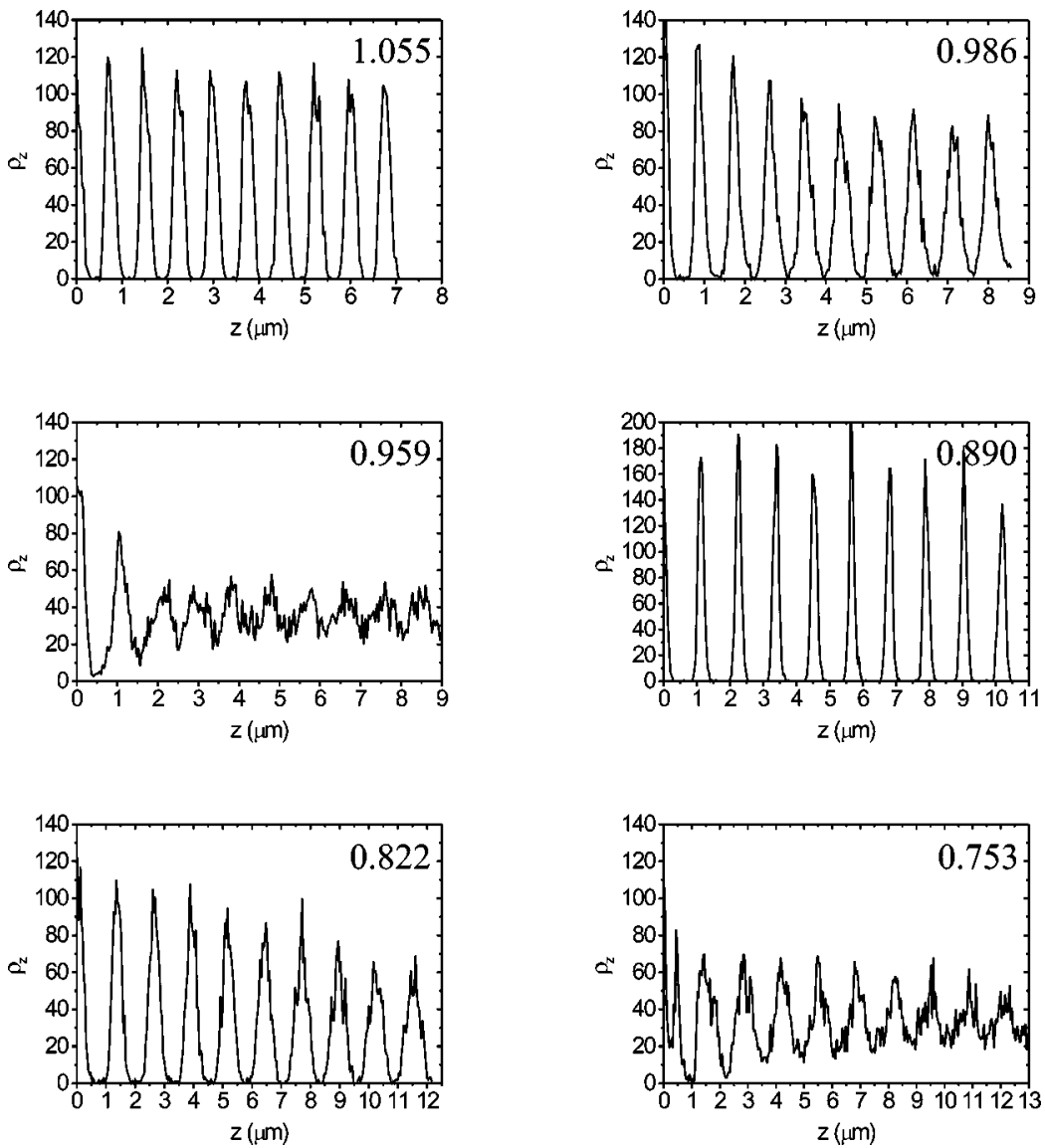

FIG. 7. The linear number density of particles perpendicular to the hcp(1100) template at different template-crystal mismatch. The values in the top right corners of every graph give the corresponding value for $a / a_{H S}$. Panels (a) and (d) correspond to "perfect" hcp and fcc crystals, respectively.

placements is found but then for the fcc crystal, as can be seen in Figs. 7(e) and 7(f). Note that in Fig. 7(f) the first layer has a split peak, indicating the formation of a new layer of upward displaced particles.

In Fig. 7 we saw a large variation in layer width and layer spacing over template-crystal mismatch, due to particle displacements and defects. In Fig. 8 it can be seen that these

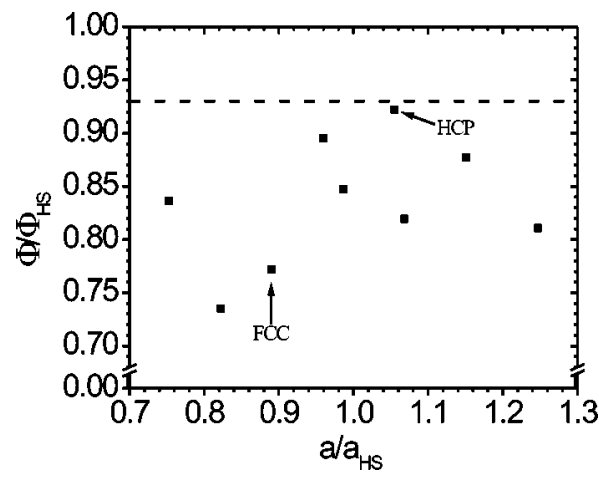

FIG. 8. Calculated volume fractions of the epitaxially grown crystals as a function of template-crystal mismatch for the isotropically stretched hcp(1100) template. The volume fractions have been scaled to the hard-sphere volume fraction given in Fig. 1, averaged over the same height in the sediment as layers 3-10. displacements also drastically influence the volume fraction calculated over layers 3-10. For the crystal at $a / a_{H S}=1.055 \mathrm{a}$ volume fraction of $\varphi / \varphi_{H S}=0.92$ is found, which is in close correspondence with the value of $\varphi / \varphi_{H S}=0.93$ that is found for the untemplated close-packed crystal. The out-of-plane displacements that occur when $a / a_{H S}$ becomes smaller lead to a strong decrease of $\varphi / \varphi_{H S}$. Remarkably, a further increase in out-of-plane displacements (see Fig. 7) leads again to an increase in volume fraction. The reason for this may be the possibility to accommodate a larger number of particles per layer at a larger layer width, through cooperative out-ofplane displacements, similar to the buckling mechanism observed in confined systems [51-53]. Note however that we do not observe the splitting of a single peak into two separate peaks, apart from that in the first layer in Fig. 7(f). The fcc crystal that grows at $a / a_{H S}=0.890$ has a volume fraction that is about 0.8 times lower than that of the hcp and the untemplated close-packed crystals.

In Fig. 9 the 2D $g(r)$ for layers 3 and 10 is shown over the same range of $a / a_{H S}$. Between the curves for $a / a_{H S}=1.055$ and $a / a_{H S}=0.986$, a small shift of all peak positions except for the first peak towards smaller distances can be seen. There are however no further qualitative differences between the forms of both curves up to at least the first ten peaks. This shift of peak positions can have two reasons. It may result from neglecting the $z$ coordinate of particles in this 

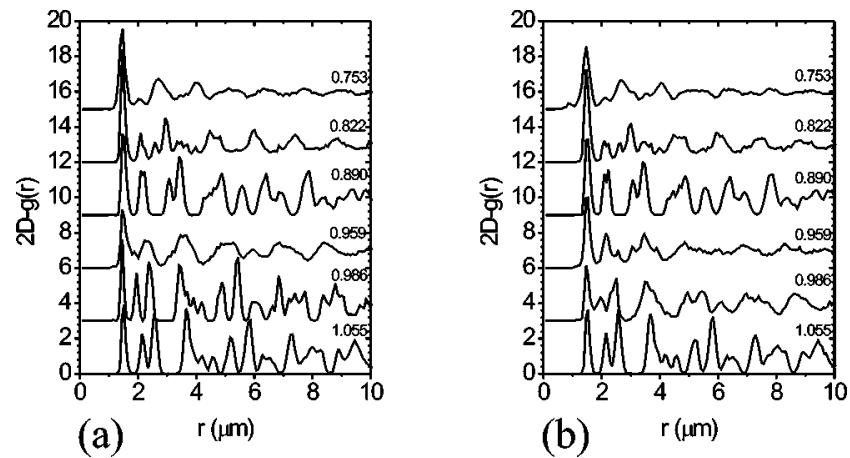

FIG. 9. In-plane (2D) radial distribution functions for the epitaxial hcp-fcc transition (a) at the third layer from the template and (b) at the tenth layer from the template. Curves for different values of $a / a_{H S}$ are shifted by 3 for clarity. Values for $a / a_{H S}$ are indicated at the right side of every curve.

layer. As we saw that the range of $z$ coordinates in a single layer widens (Fig. 7), projection of these $z$ coordinates onto a single layer will lead to an underestimation of true interparticle separations. This however would show up in a shift of all peak positions, including the first peak. A second cause may be an in-plane shift in particle positions, for instance, by a small change in the orientation of nearest neighbors. This will preserve the nearest-neighbor distance and thus the position of the first peak in 2D $g(r)$, but will change the positions of next-nearest neighbors, i.e., of all higher-order peaks. This is also what we observe for the first two curves in Fig. 9.

At $a / a_{H S}=0.959$ peak positions have smeared out and overlapped and only small, broad oscillations survive. Where initially particle displacements only caused a small shift in peak positions, here these displacements have disturbed the underlying crystal lattice so severely that even the shortrange order is not visible anymore. At $a / a_{H S}=0.890$ the long-ranged crystallinity of the fcc(100) lattice becomes visible. Note that the center position of the shifted second and third peak of the distorted hcp(1100) lattice exactly corresponds to the position of the second peak in the fcc(100) lattice. This corresponds to the transition to a more symmetric unit cell, where the four nearest neighbors have similar distances. Further shrinkage of the template unit cell again distorts first long-ranged and later short-ranged crystalline order. Between $a / a_{H S}=0.890$ and $a / a_{H S}=0.822$ particle displacements out of the second peak lead to a new peak in between second and third peaks, and particles are redistributed from the fourth peak to the joint third peak.

In Fig. 9(b), showing the 2D $g(r)$ for the tenth layer from the bottom, it can be seen whether the in-plane order at the bottom of the sample corresponds to a 3D crystalline phase or whether it is a stressed surface state slowly relaxing towards the bulk of the dispersion. The 2D $g(r)$ of the tenth layer for both the perfect hcp(1100) and fcc(100) lattices at $a / a_{H S}=1.055$ and $a / a_{H S}=0.890$, respectively, are exactly similar to that for the third layer, in peak positions as well as in peak heights. However, the shifted hcp(1100) lattice that had sharp peaks and long-ranged order in the third layer at $a / a_{H S}=0.986$ can be clearly seen to correspond to a stressed

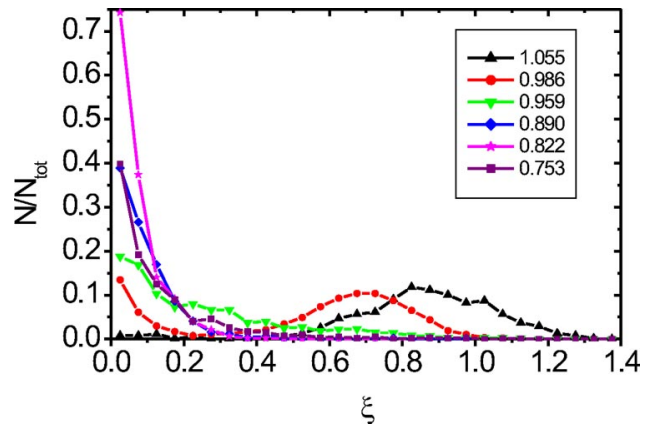

FIG. 10. (Color online) Distributions of the parameter for different values of $a / a_{H S}$ along the hcp-fcc transition. The different symbols correspond to the different values of $a / a_{H S}$ as given in the inset.

state. All peaks have broadened and overlapped and, for instance, the position of the third peak shifts back to the hcp(1100) lattice. Note however also the sharp decrease in height of the first peak, indicating that the stressed lattice expands as a whole, including nearest-neighbor distances. For the distorted fcc(100) lattice at $a / a_{H S}=0.822$, there is much less change in structure between layer 3 and layer 10 . Apparently, the fcc(100)-oriented lattice is better capable of maintaining a stressed crystalline structure. This is in accordance with visual observation with the confocal microscope where the fcc(100)-crystal structure with out-of-plane displaced grains was found to extend deep into the bulk of the dispersion (see also Ref. [24]).

In Fig. 10 the distribution of $\xi$ over particles in the fourth layer is shown. As can be seen, the shift in higher-order peak positions that was observed in the $2 \mathrm{D} g(r)$ from $a / a_{H S}$ $=1.055$ to $a / a_{H S}=0.986$ corresponds to a shift of the distribution of $\xi$ from centered at about 0.9 to centered at 0.7 . Furthermore, a small second distribution can be seen of particles that have a value of $\xi$ close to zero. The shift of the main distribution to lower values corresponds to a decreasing asymmetry of the in-plane unit cell, which corresponds to the in-plane shifts of particle positions that we identified based on the behavior of the $2 \mathrm{D} g(r)$. The distribution close to zero indicates that these shifts have transformed a small amount of unit cells to a symmetric distribution of nearest neighbors, i.e., a fcc(100) symmetry. The relative number of spheres that have a value of zero is equal to the ratio of areas under both distributions. At $a / a_{H S}=0.959$, the distribution around zero has risen and possesses a long tail that slowly decays to zero at about 0.8 . For more negative $a / a_{H S}$ a large peak at zero that sharply decays to zero remains, indicating the complete transformation to the fcc(100) lattice.

For the distribution of $\psi_{6}^{p r o j}$ (Fig. 11) the same behavior, including the initial shift of the whole distribution going from hcp(1100) to fcc(100), can be clearly observed. The widest distributions again correspond to $a / a_{H S}=0.959$ and $a / a_{H S}=0.753$, the values that showed the least pronounced translational correlations in the 2D $g(r)$.

In Fig. 12 particle coordinates in subsequent layers have been linked together into lateral displacement curves (LDC's), with all connected lateral coordinates projected onto a single $x y$ plane. In this way, it becomes visible to what 


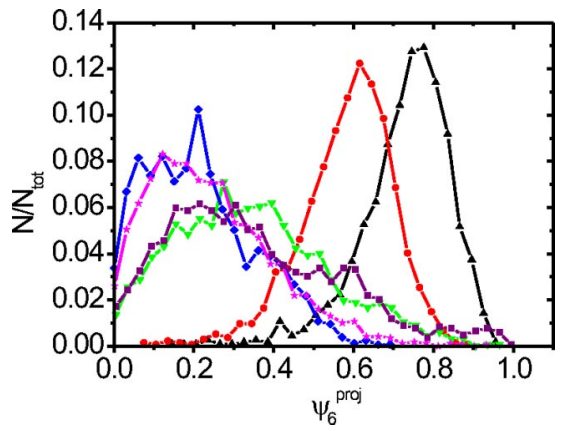

FIG. 11. (Color online) Distribution plot of the projected sixfold bond-orientational order parameter $\psi_{6}^{p r o j}$ for the hcp-fcc transition. The different symbols correspond to $a / a_{H S}=1.055$ (up-triangle), 0.986 (circle), 0.959 (down-triangle), 0.890 (diamond), 0.822 (star), and 0.753 (square).

extent order, as expressed by single-particle displacements with respect to the template, persists in the $z$ direction. In Figs. 12(a) and 10(d), the perfectly repeating lattices of both hcp(1100), giving rise to a projected hexagonal symmetry, and $\mathrm{fcc}(100)$, showing a projected square symmetry, are clearly visible. Furthermore, the shift in the orientation of nearest neighbors between $a / a_{H S}=1.055$ and $a / a_{H S}=0.986$ is also visible when comparing Figs. 12(a) and 10(b): the clear hexagons from Fig. 12(a) have been slightly distorted and a transition towards a more square symmetric lattice can already be seen. There are also small regions, such as in the bottom left corner of Fig. 12(b), where dislocations propagate in successive layers. This behavior of small regions where lateral particle coordinates are invariant over a translation in height and regions where these coordinates have a broader spread can even more clearly be seen for larger template-crystal stresses in Fig. 12(c). Thus, the initial relaxation of stress caused by a template deformation is relaxed in localized defect grains that persist in height in the sample. The fact that a single dislocation persists in height is not so surprising as this basically says that a defect or dislocation in a layer disturbs ordering in successive layers. However, what is surprising is the fact that these dislocations are grouped in localized grains and that the lateral displacements in such a defect grain seem to be directional, with the direction of successive displacements being uniform over several ten particle diameters wide [Figs. 12(c) and 10(f)], or even almost uniform throughout the entire crystal [as in Fig. 12(e)]. Note that this direction in Fig. 12(e) is along one of the fcc(100)lattice vectors.

In Fig. 13 the behavior of the root-mean-squared displacement with respect to the template, $\Delta_{n}$, is shown. First, in Fig. 13(a) the distribution of values for $\Delta$ in all layer doublets $n$ is shown. As can be seen, the distribution for the hcp crystal at $a / a_{H S}=1.055$ is sharply peaked with a maximum for $\Delta$ smaller than $0.1 \mu \mathrm{m}$. This is exactly in correspondence with what one would expect for a perfectly layered crystal and with the trajectory plot in Fig. 12(a). For smaller $a / a_{H S}$ the peak positions shifts to larger $\Delta$, showing the larger displacements of particles with respect to the template that could also be observed in Figs. 12(b) and 10(c). At $a / a_{H S}=0.959$ we can see a split distribution consisting of a sharp peak at $\Delta$
$=0 \mu \mathrm{m}$ and a broad one with a shallow peak at $\Delta$ $=0.25 \mu \mathrm{m}$ that slowly decays to zero at $\Delta=0.80 \mu \mathrm{m}$. For $a / a_{H S}=0.890$, the distribution sharpens again with the peak position shifting back to $\Delta=0.20 \mu \mathrm{m}$. Furthermore, the distribution at $\Delta=0 \mu \mathrm{m}$ has disappeared again. This illustrates the transition to the fcc lattice. Note as well that the area under the curve has become larger, showing that an increased number of particles belongs to a trajectory that can be linked to the template. This can also be seen in the inset in Fig. 13(a) where the relative number of particles that are linked in a trajectory is plotted as a function of depth in the sample.

In Fig. 13(b) $\Delta_{n}$ is shown as a function of double-layer index. Here it can be clearly seen that the sharp distribution with averaged value of $\langle\Delta\rangle=0.1 \mu \mathrm{m}$ that we observed for $a / a_{H S}=1.055$ corresponds entirely to lattice vibrations as its averaged value is constant throughout the crystal. Relative to the mean interparticle distance this value $\langle\Delta\rangle / R_{H S} \approx 0.07$, which is well below the Lindemann criterion that states that the relative mean-squared displacement has a value of 0.15 at melting. A similar situation can be observed for $a / a_{H S}$ $=0.890$, corresponding to the (100)-oriented fcc crystal. Thus we can conclude that the larger offset of $\langle\Delta\rangle=0.2 \mu \mathrm{m}$ is due to the mismatch of the fcc(100) lattice with the template. For all other values of $a / a_{H S},\langle\Delta\rangle$ increases as a function of depth in the sample. Note that $n=5$ corresponds to eight layers deep. For $a / a_{H S}=0.986$, we see that in the bottom of the sample $\langle\Delta\rangle$ starts at $0.1 \mu \mathrm{m}$, but starts slowly increasing after the first six layers due to the relaxation of the stressed template positions. For the other values of $a / a_{H S}$, this also happens starting from the fcc value of $\Delta=0.2 \mu \mathrm{m}$, where for the two smallest values of $a / a_{H S}$ the curves are rapidly diverging.

\section{Positive isotropic deformations of the hcp(1100) lattice}

The same analysis that was performed above on the structural evolution over negative isotropic deformations of the hcp(1100) template can be carried out over positive, stretched, deformations. The behavior of the linear number density as function of $a / a_{H S}$ (not shown) is similar to that shown for negative deformations: starting from a perfectly layered crystal, peaks gradually broaden due to out-of-plane displacements and again overlap, leading to a situation with a constant offset density with small oscillations for $a / a_{H S}$ $=1.397$. For this highest value of $a / a_{H S}$, the layers are not well defined anymore after eight layers, as the oscillations decay and only a constant density with single-binsize wide noise peaks remains. Both for the isotropic expansion as well as for isotropic shrinkage of the template unit cell around the optimal value, there is a sharp decrease in $\varphi / \varphi_{H S}$, followed again by an increase when the number of out-of-plane displacements further increases. For $a / a_{H S}=1.397$, the layer spacing of layers 3-8 was extrapolated to find the position of a "tenth" layer for comparison with the other results.

In Fig. 14 the in-plane 2D $g(r)$ for the third and for the tenth layer above the template is given, again starting with $a / a_{H S}=1.055$, the value that corresponds to hep-crystal growth. Already at $a / a_{H S}=1.068$ the effect of the expanded template can be seen, resulting in displacements of a small 
(a)

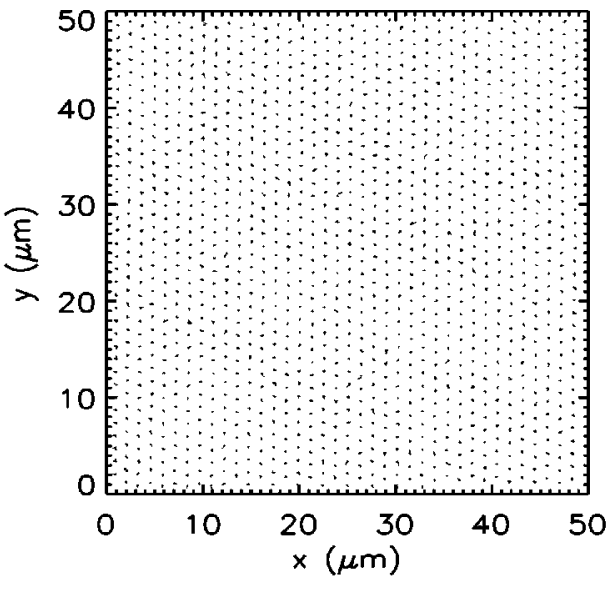

(c)

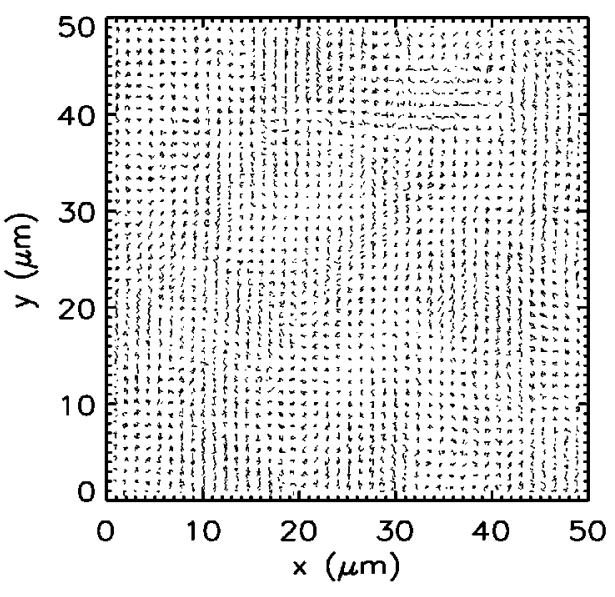

(e)

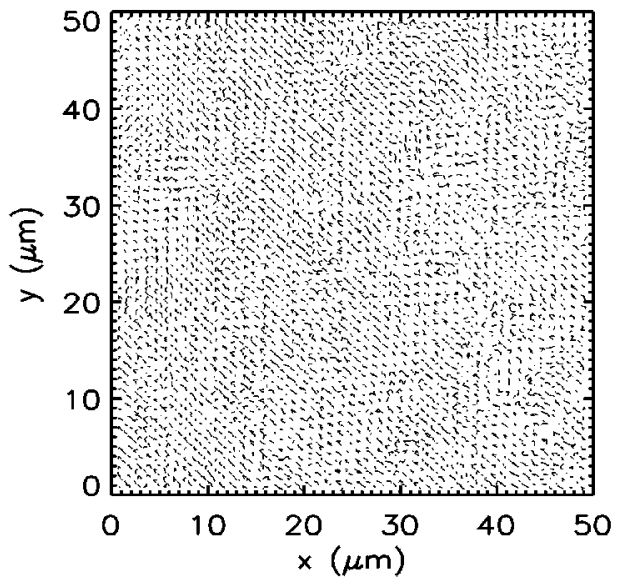

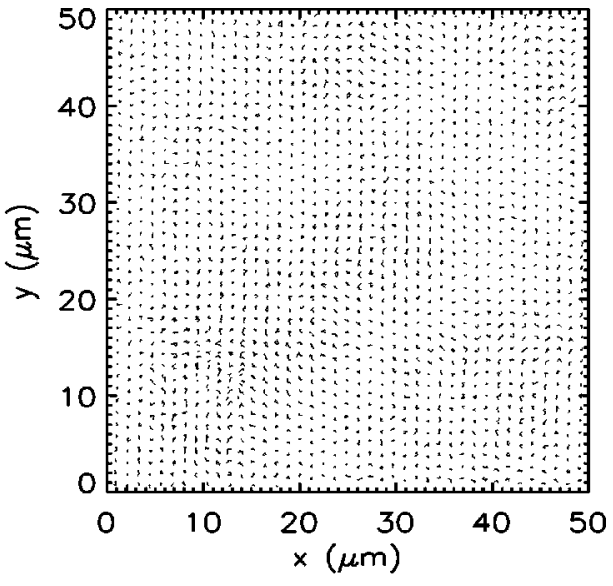

(d)
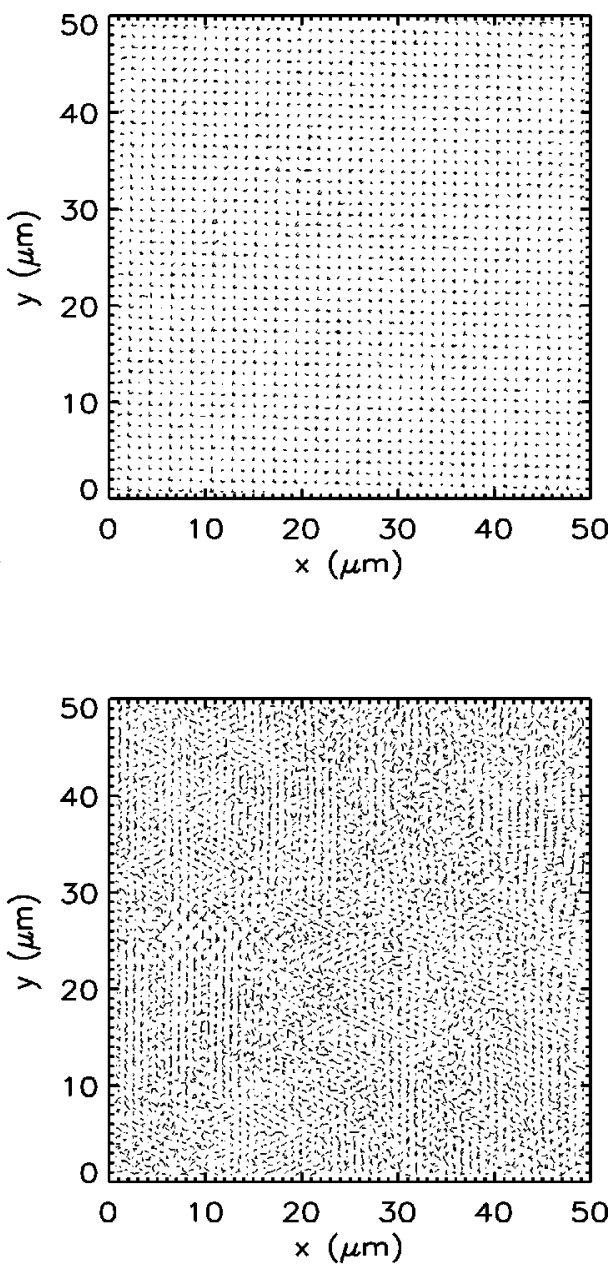

FIG. 12. Lateral displacement curves (LDC's) of particles in the first 20 layers above the template. (a) $a / a_{H S}=1.055$, (b) $a / a_{H S}$ $=0.989$, (c) $a / a_{H S}=0.959$, (d) $a / a_{H S}=0.890$, (e) $a / a_{H S}=0.822$, (f) $a / a_{H S}=0.753$. Panels (a) and (d) again correspond to perfect hcp and fcc crystals, respectively. In the other panels directional shifts in particle positions due to stress relaxation are visible.

amount of particles towards larger particle-particle separations than for $a / a_{H S}=1.055$ : the second curve from the bottom shows peak broadening to larger distances, especially in the small extra "kinks" in the first and third maxima. Fur- thermore, the density in between second and third peaks increases. At $a / a_{H S}=1.151$ this effect is more pronounced: the second and third peaks almost completely overlap and the long-ranged correlations disappear. At the largest isotropic 

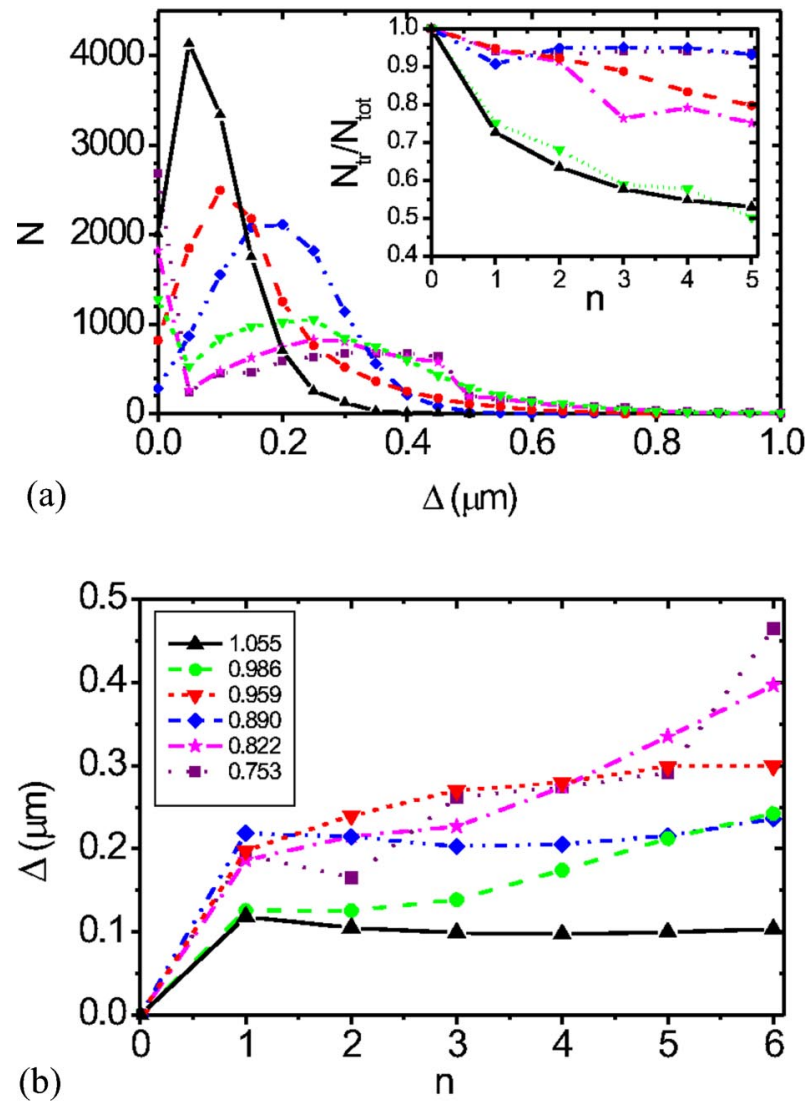

FIG. 13. (Color online) (a) Distribution of the root-meansquared $x y$ displacements $\Delta$ of particles in successive layers. The inset shows the relative number of particles contained in LDC's as a function of two-layer index $n$. The different lines correspond to $a / a_{H S}=1.055$ (solid), 0.986 (dash), 0.959 (short dash), 0.890 (dashdot-dot), 0.822 (dash-dot), and 0.753 (dot). (b) The averaged $\Delta$ as a function of $n$. The symbols correspond to different values of $a / a_{H S}$ as given in the inset. Lines connecting data for each $a / a_{H S}$ have been drawn to guide the eye, where the line type for each $a / a_{H S}$ corresponds to that in (a).

deformation of $a / a_{H S}=1.397$, sharp peaks are again coming up, but their relative positions do not seem to correspond anymore to the $h c p(1100)$ lattice.

When looking at Fig. 14(b), it can be seen that the stressed structure in the bottom layers relaxes back to the $\mathrm{hcp}(1100)$ lattice when moving further away from the template. Even at $a / a_{H S}=1.151$, the translational correlations in the $2 \mathrm{D} g(r)$ of the tenth layer correspond to those of the hcp(1100) lattice over up to $10 \mu \mathrm{m}$, indicating the possibility for rearrangements from the stressed surface layers.

The behavior of the $\xi$ parameter (Fig. 15) is consistent with the behavior described above. Initially, at $a / a_{H S}$ $=1.068$ the distribution broadens, indicating small particle displacements from the hcp(1100) lattice. Then, for larger $a / a_{H S}$, the distribution shifts to higher values of $\xi$. Thus, though the deformation of the unit cell is isotropic, the distribution of nearest-neighbor distances becomes more asymmetric. In fact, in Fig. 14 it can be seen that the nearest neighbor distance remains constant throughout the deformations, so the positions of next-nearest neighbors shift rela-
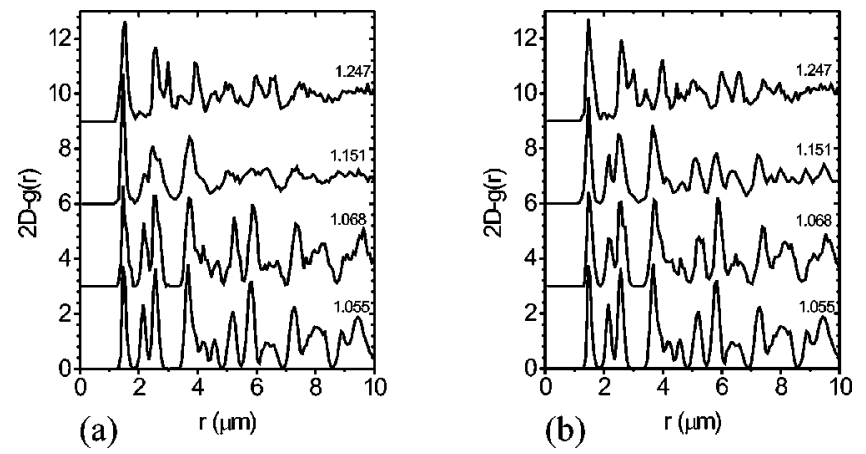

FIG. 14. In-plane (2D) radial distribution functions for the isotropically stretched hcp template (a) at the fourth layer from the template and (b) at the tenth layer from the template. Curves for different values of $\varepsilon$ are shifted by 3 for clarity. Values for $a / a_{H S}$ are given on the right side of each curve.

tively more with the deformed lattice than do nearest neighbors. As can be seen this shift of the distribution sets through, while meanwhile also a peak at zero develops corresponding to defected regions with a close-packed unit cell. The relaxation towards the hcp(1100) lattice at especially the template with $a / a_{H S}=1.151$ can also be clearly seen when comparing the distributions for third and tenth layers.

In Fig. 16 it can be seen that the deformed nearestneighbor distributions still maintain a strong hexagonal symmetry in a two-layer projection up to $a / a_{H S}=1.151$. Although the decay towards zero becomes of longer range, the main

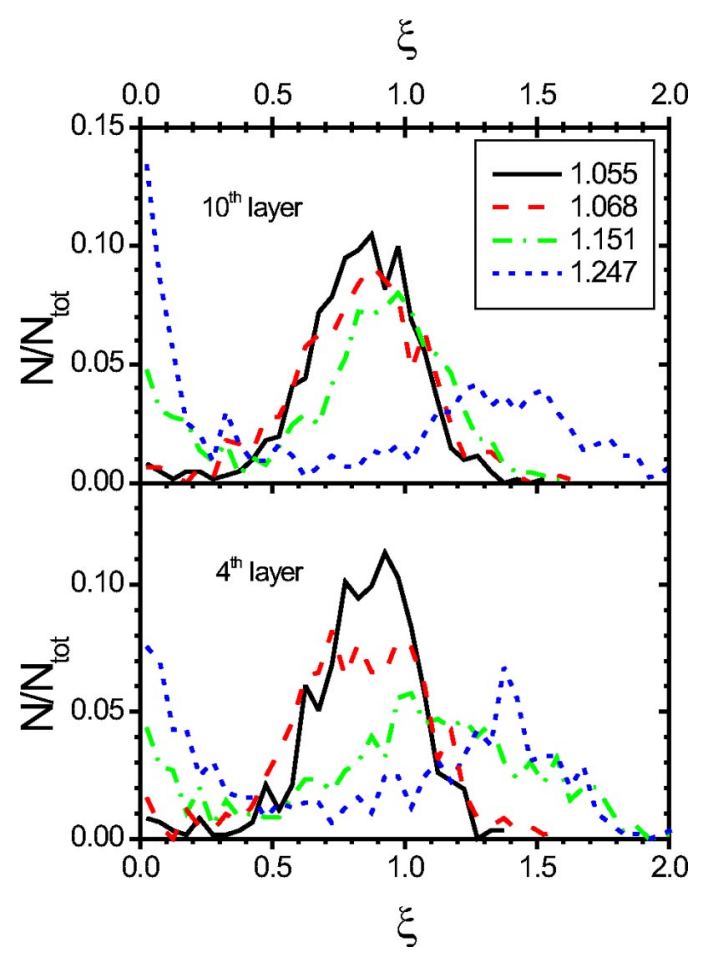

FIG. 15. (Color online) Distributions of the $\xi$ parameter for different values of $a / a_{H S}$ for a stretched hcp template. The lower curves give values for the fourth layer in the sample; the upper curves for the tenth layer in the sample. Different line types correspond to the different values for $a / a_{H S}$ as given in the inset. 


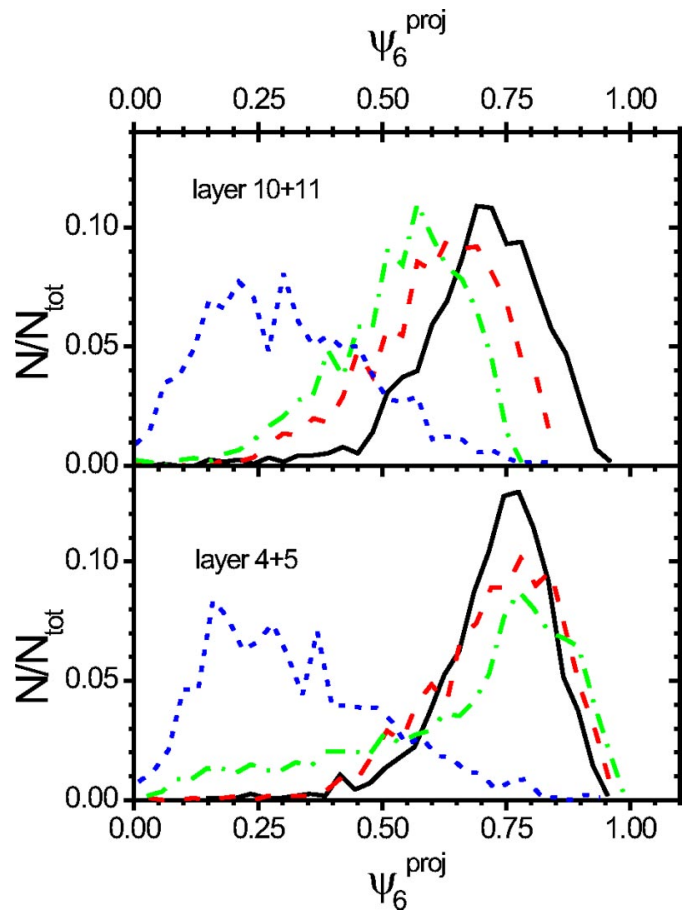

FIG. 16. (Color online) Distribution plot of $\psi_{6}^{\text {proj }}$ for the stretched hcp template for a projection of third and fourth layers (lower curves) and 10th and 11th layers (upper curves). The different lines correspond to $a / a_{H S}=1.055$ (solid), 1.068 (dash), 1.151 (dash-dot), and 1.397 (short dash).

distribution remains centered around 0.8 , corresponding to the hcp(1100) lattice. Also indicative of a relaxation process is the flattening of the root-mean-squared displacements with respect to the template (Fig. 17) for $a / a_{H S}=1.068$ at $n=3$, corresponding to the fourth layer, to a value of $\Delta=0.2 \mu \mathrm{m}$. For $a / a_{H S}=1.151$ the curve seems to be slowly converging as well, though a thicker crystal has to be examined to clarify whether this is actually the case. For $a / a_{H S}=1.397$ the relative number of particles contained in LDC's is already below 0.6 at $n=2$ (not shown) and, as mentioned before, after eight layers it is not possible anymore to distinguish individual layers. In fact the distribution of values for $\Delta$ showed a single peak at zero (not shown), such as the first peak in the

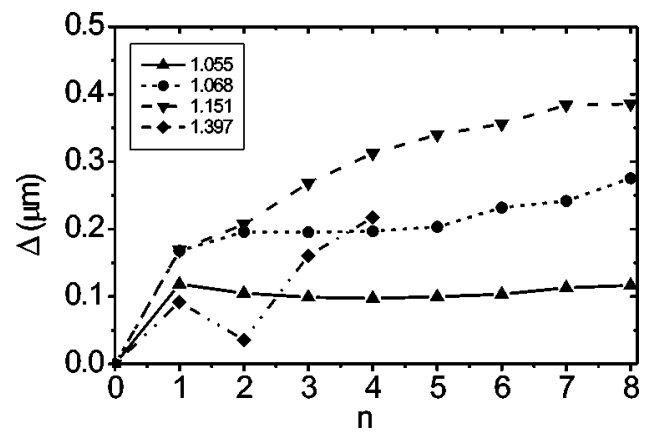

FIG. 17. Root-mean-squared displacements of particles relative to the template as a function of double-layer index for different values of $a / a_{H S}$ for the stretched hcp(1100) template.

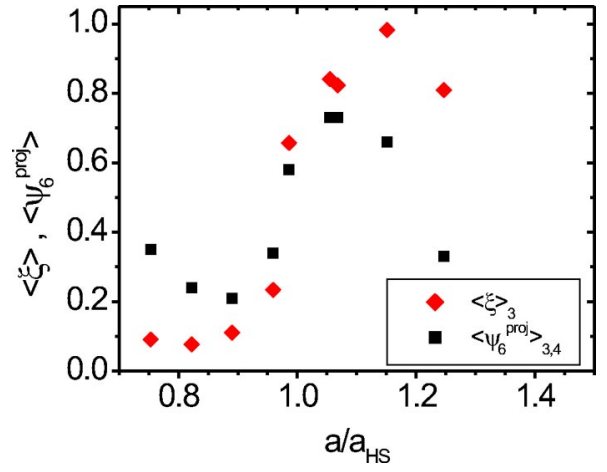

FIG. 18. (Color online) The development of both the layeraveraged values for the order parameters $\xi$ (in layer 3 ) and $\psi_{6}^{\text {proj }}$ (in layer 3 and 4) as a function of template-crystal mismatch for the isotropically stretched templates.

distributions for the lowest values of $\varepsilon$ in Fig. 13(a), indicating that almost all of these LDC's consist of uncorrelated positions.

Finally, in Fig. 18 the values for both $\langle\xi\rangle_{3}$ and $\left\langle\psi_{6}^{\text {proj }}\right\rangle_{3,4}$ are shown for all of the isotropically stretched templates, both positive and negative. Here, the influence of the deformations of the template on both order parameters can be clearly seen: the $\xi$ parameter follows the direction of the deformation, becoming larger for stretching the template and smaller for shrinking, while $\psi_{6}^{p r o j}$ indicates the disturbance of in-plane symmetry when the template is deformed with respect to $a / a_{H S}=1.055$. Figure 19 shows two examples of structures that were denoted as disordered according to the criteria given before. As can be seen for the isotropic deformation with $a / a_{H S}=1.247$, the structure of the template is still locally visible, but with a considerable amount of defects, including "stacking faults" which destroy local symmetry and translational order. At a template deformation with $a / a_{H S}=1.40$ and $c / c_{H S}=1.23$, no effect of the template other than absence of ordering is visible anymore.

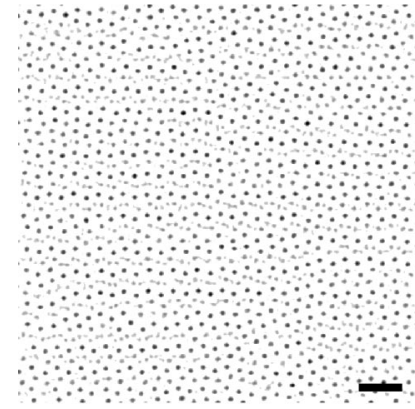

(a)

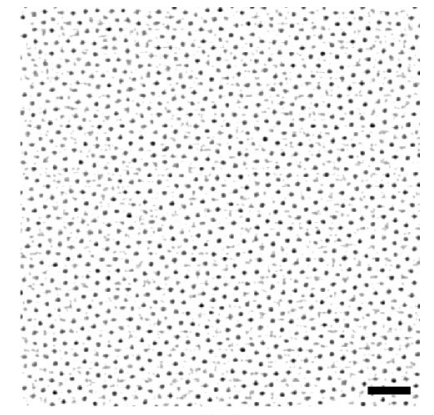

(b)
FIG. 19. Confocal microscopy images of colloidal structures that were qualified as disordered in this study (a) At a height of $3 \mu \mathrm{m}$ above a template with $a / a_{H S}=1.247$. Structuring in the template direction is still visible, but with a lot of defects and height differences (vaguely visible out-of-focus particles). (b) At a height of $1.3 \mu \mathrm{m}$ above a template with $a / a_{H S}=1.40$ and $c / c_{H S}=1.23$. In both images the scale bar indicates $5 \mu \mathrm{m}$. 


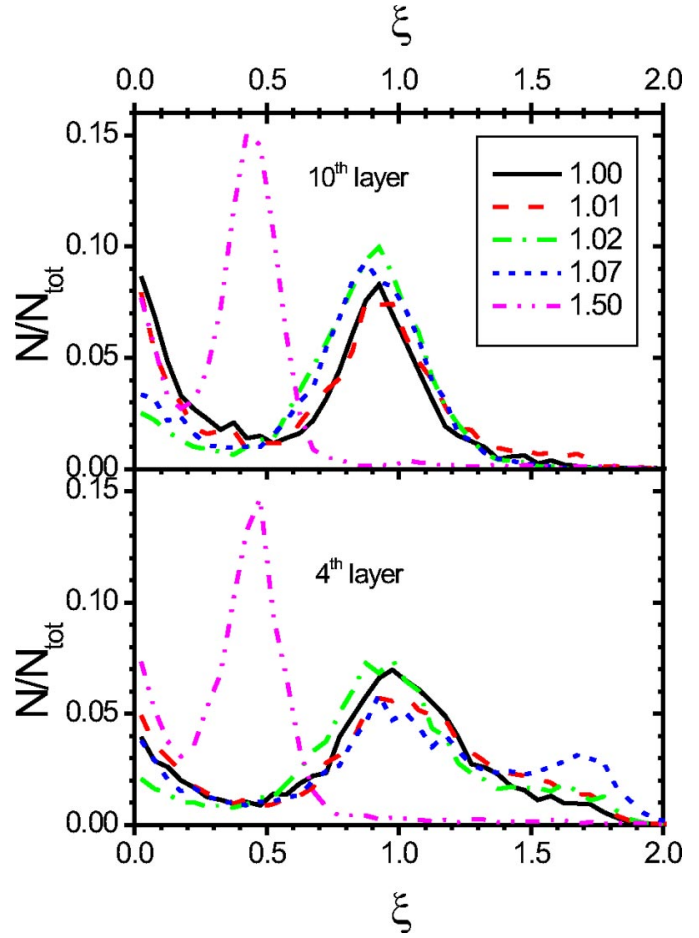

FIG. 20. (Color online). The distribution of the $\xi$ parameter for the anisotropically stretched template with $a / a_{H S}=1.076$. The different line types correspond to the different values of the axis ratio, $\left(c / c_{H S}\right)\left(a / a_{H S}\right)^{-1}$, as given in the inset. The lower curves correspond to the fourth layer; the upper curves to the tenth layer.

\section{Anisotropic (c/a) deformations}

Starting from two points for the isotropically stretched template, an anisotropic stretch of the template in the direction of the $c$ axis was performed. The structural evolution for these deformations is given in Fig. 5. Both stretches were performed starting from a unit cell with $\left(a / a_{H S}, c / c_{H S}\right)$ values slightly off the regime that was identified as the best for growing a hcp crystal, namely, at $\left(a / a_{H S}, c / c_{H S}\right)$ $=(0.99,0.99)$ and at $\left(a / a_{H S}, c / c_{H S}\right)=(1.08,1.08)$. Starting from the shrunken unit cell, the hcp crystal remains stable up to $c / c_{H S}=1.05 a / a_{H S}$, after which (111)-oriented crystals grow over the template. From the stretched template the stability regime is larger, up to $c / c_{H S}=1.15 a / a_{H S}$, leading at $c / c_{H S}=1.5$ to a new supercrystal structure that we will treat in the following section. For templates with a larger stretch, a (111)-oriented crystal nucleates in this case as well. For both series, an anisotropic stretch of the template unit cell with $c / c_{H S}=1.02$ leads to better hcp crystal quality than for $c / c_{H S}=1.0$ and $c / c_{H S}=1.01$. As this behavior was found to be similar for both series, we will limit ourselves here to the series starting from $\left(a / a_{H S}, c / c_{H S}\right)=(1.08,1.08)$.

In Fig. 20 the $\xi$ parameter for five different values of $\left(c / c_{H S}\right)\left(a / a_{H S}\right)^{-1}$ is given. The bottom curves correspond to the fourth layer from the bottom and the upper curves for the tenth layer. As can be seen, all curves for $c / c_{H S}=1.0 a / a_{H S}$ to $1.07 a / a_{H S}$ have a main distribution with a maximum slightly below $\xi=1.0$, corresponding to what was seen before for a template stretched compared to the ideal values, together

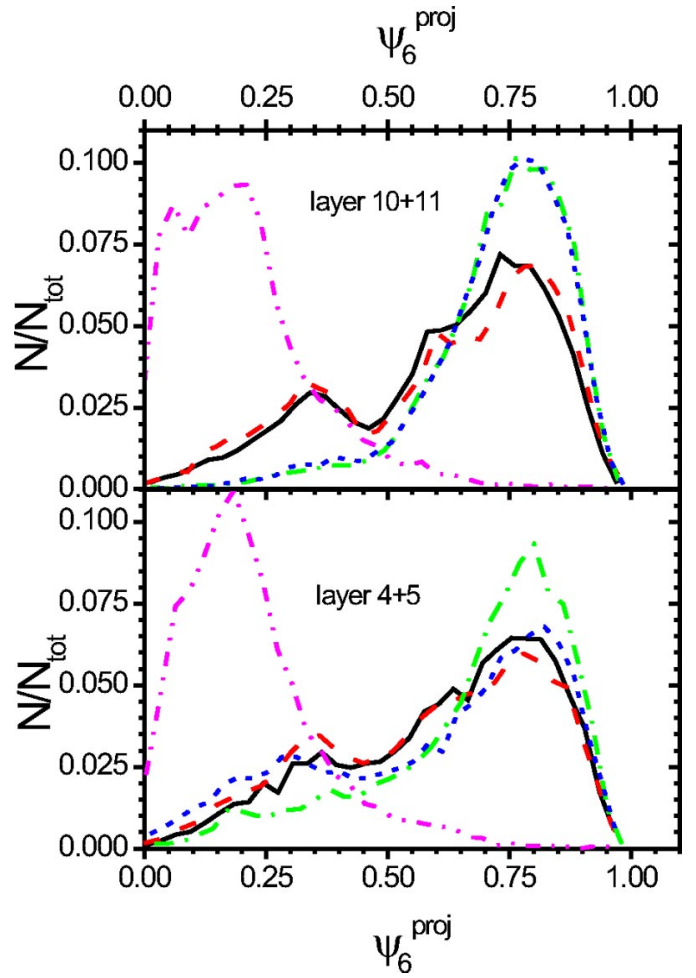

FIG. 21. (Color online) Distribution plots for $\psi_{6}^{p r o j}$ for the anisotropically stretched template with $a / a_{H S}=1.076$ for a projection of fourth and fifth layers (lower curves) and 10th and 11th layers (upper curves). Different line types correspond to axis ratios of $\left(c / c_{H S}\right)\left(a / a_{H S}\right)^{-1}=1.00$ (solid line), 1.01 (dash), 1.02 (dash-dot), 1.07 (short dash), and 1.50 (dash-dot-dot), respectively.

with a smaller distribution of values close to 0.0 , indicating the presence of defects. For the fourth layer, the maximum of the distribution decreases going from $c / c_{H S}=1.0 a / a_{H S}$ to $1.01 a / a_{H S}$, but at $c / c_{H S}=1.02 a / a_{H S}$ increases again and shifts from 0.95 to a slightly smaller value of 0.85 . Furthermore, the distribution at $\xi=0$, and thus the number of defects, decreases. This behavior is even more pronounced in the tenth layer. Where for $c / c_{H S}=1.0 a / a_{H S}$ and $1.01 a / a_{H S}$ the maximum of the distribution at $\xi=0$ has doubled relative to the fourth layer, this is not the case for $c / c_{H S}$ $=1.02 \mathrm{a} / \mathrm{a}_{H S}$ and even $1.07 \mathrm{a} / \mathrm{a}_{H S}$. The increase of peak height at $\xi=1.0$ and the shift of this distribution towards slightly smaller values has also become more pronounced. For all four curves this distribution has sharpened, indicating that the distribution of nearest-neighbor distances for particles where this distribution is hcp(1100)-like has relaxed from the stressed stretched unit cell of the template towards ideal hcp(1100) higher in the sample. This change is more pronounced for $c / c_{H S}=1.02 a / a_{H S}$ and $1.07 a / a_{H S}$ than for $c / c_{H S}=1.0 a / a_{H S}$ and $1.01 a / a_{H S}$. For $c / c_{H S}=1.07 a / a_{H S}$ the small distribution of large asymmetries at $\xi=1.7$ in the fourth layer has disappeared in layer 10.

The distribution of $\psi_{6}^{p r o j}$ for layers 3 and 4 and layers 10 and 11 are shown in the lower and upper parts of Fig. 21, respectively. Here, the increase in crystalline order for $c / c_{H S}=1.02 a / a_{H S}$ compared to $1.0 a / a_{H S}$ and $1.01 a / a_{H S}$ is even more clearly visible. Already in layers 3 and 4 the curve 


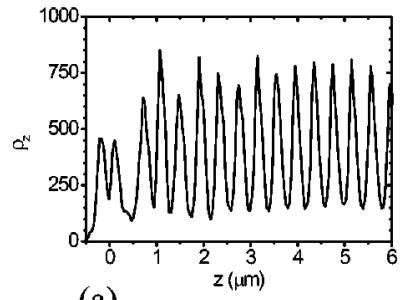

(a)

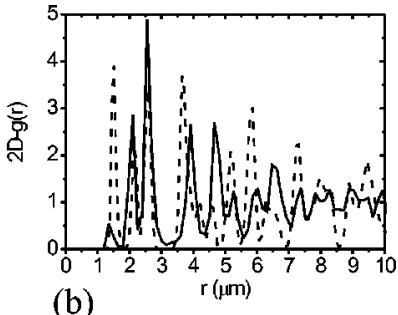

(b)

FIG. 22. (a) Linear number density perpendicular to the template for a crystal at an anisotropically stretched template with $a / a_{H S}=1.076$ and an axis ratio of $\left(c / c_{H S}\right)\left(a / a_{H S}\right)^{-1}=1.50$.

for $c / c_{\mathrm{HS}}=1.02 a / a_{H S}$ has a 1.5 times higher maximum than for $c / c_{H S}=1.0 a / a_{H S}$ and $1.01 a / a_{H S}$, while the small shoulder at $\psi_{6}^{\text {proj }}=0.35$ has disappeared. In layers 10 and 11 these differences are even better visible. Furthermore, the large increase in hcp crystal order higher in the sample for $c / c_{H S}$ $=1.07 a / a_{H S}$ is also clearly evident: not only has the maximum value increased to almost 0.1 , similar to the relaxation at $c / c_{H S}=1.02 a / a_{H S}$, the shoulder at $\psi_{6}^{p r o j}=0.35$ that has become more pronounced for $c / c_{H S}=1.0 a / a_{H S}$ and $1.01 a / a_{H S}$ has completely disappeared for $c / c_{H S}=1.07 a / a_{H S}$.

\section{Non-close packed HS crystal}

In Fig. 20 it can be seen that at $c / c_{H S}=1.50 a / a_{H S}$ the distribution of $\xi$ contains a remarkably sharp and, compared to the hcp distributions also narrow, distribution centered at 0.5 . This strongly indicates the emergence of a structure with a replicating unit cell that has half the asymmetry in nearestneighbor distances than has the hcp(1100) unit cell. The distribution of $\psi_{6}^{p r o j}$ in Fig. 21 shows a distinct peaked distribution as well, here with a maximum at $\psi_{6}^{p r o j}=0.20$. The behavior of these two order parameters is different from that of the other crystalline structures that we encountered over the hcp(1100) template before, such as fcc(100) (see Figs. 10 and 11) and hexagonal.

In Fig. 22(a) $\rho_{z}$ for the structure on this template is shown. The structure consists of sharp layers, with a peak width comparable to that of the hcp and fcc crystal in Fig. 7. The distance between the peaks has a constant value of $\Delta z$ $=0.50 \pm 0.01 \mu \mathrm{m}$. The constant "offset" density of about 0.15 times the average peak height is caused by small defect grains that were found to coexist with this structure (also note the distribution of $\xi$ at 0 in Fig. 20). The fact that there is a split first peak is probably caused by the fluorescence of the template which makes the determination of particle positions close to the template harder. The small interlayer spacing of $0.50 \mu \mathrm{m}$ suggests a low in-plane density. In fact, if we look at the 2D $g(r)$ in Fig. 22(b), it can be seen that the first peak of the hcp(1100) lattice is almost completely absent. Thus the mean interparticle distance has increased towards a value of about $2 \mu \mathrm{m}$, showing that indeed the inplane density is lower than for the close-packed planes. The first two peaks in 2D $g(r)$ actually fall on the same positions as the second and third peaks in the hcp(1100)-2D $g(r)$. For larger distances peak positions in both functions slowly run out of registry with each other.
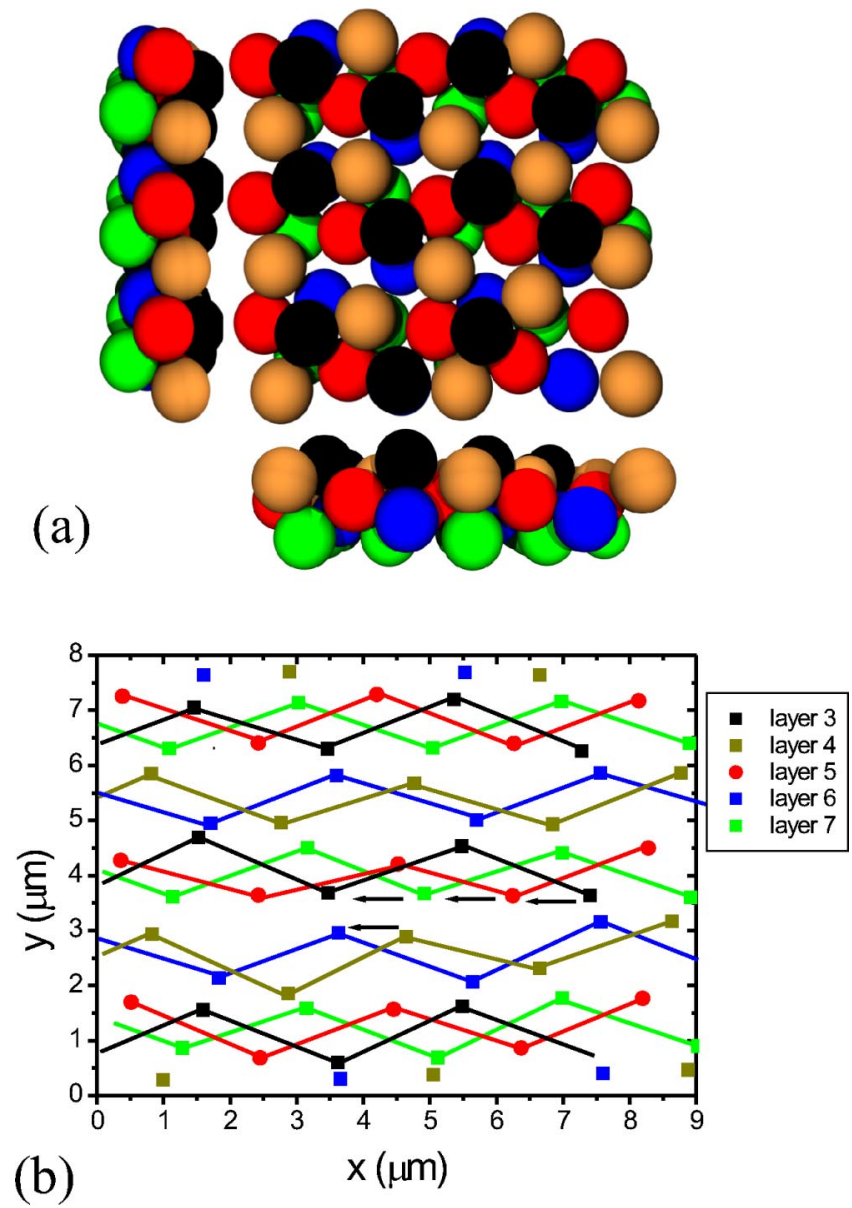

FIG. 23. (Color online) (a) Image showing particle coordinates in a small part of our real-space data set in layers 3-7 for the crystal at an anisotropically stretched template with $a / a_{H S}=1.076$ and an axis ratio of $\left(c / c_{H S}\right)\left(a / a_{H S}\right)^{-1}=1.50$. The image in the middle shows a top view, the other two the respective side views. Spheres, with diameter drawn to scale, have been colored corresponding to their layer index: green for layer 3, blue for layer 4, red for layer 5 , gold for layer 6, and black for layer 7. (b) A projection of $x y$ particle coordinates for the spheres (a) onto a single layer. The symbols corresponding to the different layers are given in the legend next to the graph. Positions indicated with filled symbols have been connected with solid lines and open symbols with dashed lines, in order to indicate the template-induced alignment. The arrows indicate the shift in particle positions with the original template $A B A$ sequence between layers $3,5,7$, and $9(=3)$ and layers 4 and 6 , respectively.

In Fig. 23(a) an image of a small part of the crystal is shown with spheres drawn at the determined $x y z$ positions. Both the small interlayer spacing as well as the open in-plane structure are clearly visible in this image. Figure 23(b) shows the $x y$ coordinates for particles in Fig. 23(a) projected onto a single plane. The positions in successive layers exactly follow the zigzag of the stretched hcp(1100) lattice with the coordinates for particles in layers $i$ and $i+1$ falling in different "lanes" displaced in the $a$-axis direction. The extra space created by stretching the template in the $c$ direction is accommodated by shifting positions in layers $i$ and $i+2$ within these lanes by $1 \mu \mathrm{m}$, as indicated with the arrows drawn in Fig. 23(b). These shifts indicate that the structure should be 
periodic when translated over six layers, and indeed we have found that the particle positions in the ninth layer fall on top of those in layer 3 .

For the hard-sphere scaled volume fraction of this crystal we found a value of $\varphi / \varphi_{H S}=0.78$, compared to $\varphi / \varphi_{H S}$ $=0.92$ for the crystal grown on a templateless part of the bottom wall. This value is however higher than that for the best hcp crystal in this series of anisotropic deformations, namely, that at $c / c_{H S}=1.02 a / a_{H S}$, which was $\varphi / \varphi_{H S}=0.74$. This again highlights that the distorted crystals that grow at the deformed templates are, strictly speaking, not close packed as well. As noted before and visible in Figs. 20 and 21 the non-close-packed superstructure alternates with defect grains. This can be due to the fact that our deformed hcp(1100) template is not the best suited template to grow this crystal structure. The most obvious choice for the "ideal" template would seem to be a template that has an ansotropic stretch of the axis ratio of 1.50, such as in this case, but starting from the ideal hcp(1100) plane or, as there may again be prefreezing or wetting, starting from the hcp(1100) template that generated the best quality hcp crystal. It would certainly be interesting to further examine the growth conditions for this peculiar hard-sphere crystal structure.

\section{SUMMARY AND DISCUSSION}

In this paper we have demonstrated the possibility to grow crystal structures that are metastable in bulk crystallization using colloidal epitaxy. For colloids interacting through a hard-sphere-like potential, a hcp(1100) pattern was found to induce hcp crystal formation. Modification of this surface pattern gives the possibility to directly dictate the crystal's stacking sequence, which was illustrated by growth of a "double hcp" (dhcp) crystal.

For the hcp(1100) template the evolution of crystal structure over template-crystal mismatch was studied using a 3D real-space analysis. The first step in our analysis consisted of examining the degree of layering parallel to the template. A template-crystal mismatch was found to lead to out-of plane displacements of particles, in accordance with observations in molecular epitaxy [41,42]. Thus in further research on colloidal epitaxy, the width of peaks and/or the relative depth of minima in the linear number density perpendicular to the template may be used as parameters for probing the quality of the epitaxially grown crystal and for identifying the best conditions for epitaxial crystal growth. Relatively small values for the layer width and an interlayer density of essentially zero correlate with sharp peaks and long-ranged inplane order as evidenced in a $2 \mathrm{D} g(r)$ and with the indications given by the other parameters that we have used. For the hcp crystal these conditions were met at an isotropically stretched template with lattice parameters $a / a_{H S}$ $=c / c_{H S}=1.055$. Scaling the template isotropically from these values led to the out-of-plane displacements mentioned above. For a template where the unit-cell dimensions were shrunken to $a / a_{H S}=0.89$, there was a structural transition to a (100)-oriented fcc crystal. Further shrinkage as well as large isotropic stretches led to disorder in the first layers over the template. The volume fraction of the epitaxially grown crys- tal was found to vary with template-crystal mismatch, with the highest volume fraction crystal growing at the isotropically stretched template.

For a hard-sphere system in zero gravity, Heni and Löwen demonstrated that nonhexagonal surface patterns prefreeze and/or wet at a unit cell of which both lattice spacings are stretched by a factor of 1.035 [38,39]. This may be readily explained by the fact that a solution crystallizing at lower volume fractions than bulk freezing has correspondingly larger lattice parameters than for a crystal at bulk volume fractions. Thus our result that an isotropically stretched template grows the best quality hcp crystal may be an indication that the hcp(1100) shows prefreezing or even wetting. Recently, Pronk and Frenkel have shown that a deformation of the hard-sphere crystal structure, such as the one implied by our template, changes the relative stability of hcp and fcc crystal structures [40]. The difference however remains small, on the order of $10^{-2} k_{B} T$ per particle in favor of hcp.

For the isotropically stretched template we also found the highest volume fraction, which was furthermore almost similar to the volume fraction of the untemplated close-packed crystal. This last fact is not in correspondence with what one would expect for a stretched unit cell. The reason why this higher volume fraction was reached was that the distance between the hcp(1100) planes in this crystal was smaller than what would be expected for the stretched hcp(1100)-lattice vectors. This is probably caused by compression of the crystal when more particles sediment and crystallize on top of it: at first a few layers wet or prefreeze at the stretched template, but when during sedimentation the pressure increases, the crystal cannot isotropically compact as decreasing the inplane lattice vectors would result in an increase in interlayer distance opposite to the gravitational field. Thus the stretched in-plane lattice vectors are preserved and the interlayer distance in the crystal is smaller to accommodate the higher volume fraction. For the (100)-oriented fcc crystal an approximately 0.8 times lower volume fraction than that of the hcp crystal was found, which was also lower than that for any of the other defected hcp crystals. The quality of the crystal, as judged from the behavior of the various order parameters used, was however comparable to that of the best hcp crystal. Furthermore, the in-plane nearest-neighbor distance did not noticeably change between the hcp and the fcc crystal, indicating that the fcc crystal also showed prefreezing or wetting. Apparently, the (1100)-oriented hcp crystal can be compressed much more to accommodate the increasing osmotic pressure than the (100)-oriented fcc crystal. Indeed, for the fcc crystal we found a layer spacing that was a factor 1.11 higher than the expected value based on the effective hard-sphere radius, while for the (1100)-oriented hcp crystal these two values corresponded. Pronk and Frenkel found that the elastic constants of fcc and hep crystals can be surprisingly different, with differences up to $20 \%$ [40]. The deformations and compressions reported here are however far beyond the scope of their calculations.

The dependence of volume fraction on template-crystal mismatch starting from the stretched template that yielded the highest volume fraction and best quality crystal was as follows: A small deviation of lattice parameters from the ideal ones led to a sharp decrease in volume fraction after 
which there was again a rise in volume fraction when the layer width further increased. The reason for this may be the possibility to accommodate a larger number of particles per layer at a larger layer width, through cooperative out-ofplane displacements, similar to the buckling mechanism observed in confined systems [51-53].

Based on layerwise defined order parameters the structural evolution was examined in more detail. This analysis showed the structural relaxation in subsequent layers above a mismatch template. However, it should be noted that the sample used in the experiment with isotropically shrunken and expanded unit cells consisted of only thin crystals, with a sediment thickness of about $20 \mu \mathrm{m}$. As such the osmotic pressure at the tenth layer is considerably lower than at the template. This however hardly changed the calculated hardsphere volume fraction calculated over the range in $z$ spanned by the first ten layers. Furthermore, in the experiments with the anisotropically stretched templates a higher initial volume fraction was used, which resulted, over the range of layers examined, in a constant volume fraction of almost 0.74 corresponding to complete compaction. In these crystals similar relaxation and rearrangements as a function of height were still observed. It is known from molecular epitaxy that structural transitions can take place at over 20 layers above a mismatch substrate [54]. For a system of density-matched colloids, we have recently found that a stressed surface crystal can extend for over 30 layers deep [24]. The dependence and extent of epitaxial crystal structure on sediment thickness need to be explored in more detail in further research, both for the mismatch structures as well as for the "perfect" epitaxial crystals. This will also give additional insight into the strength of the entropic interaction of the templated wall with the hard-sphere system.

By analyzing particle positions in successive layers with respect to template and to each other, and thus constructing lateral $(x y)$ displacements curves (LDC's), the location of and correlations between dislocations and defects were studied. The root-mean-squared displacements in these LDC's provided a sensitive order parameter for crystal quality. For the hcp crystal these were found to be constant at 0.14 times the particle radius and for the fcc crystal constant at 0.28 times the particle radius. For small mismatches these values increase and diverge higher in the crystal. Furthermore, these trajectory plots give insight in correlated displacements of particle due to surface strain. At increasing mismatch and thus increasing particle displacements with respect to perfect crystal positions there is a structural breakup in crystal grains and defect grains, with these displacements being localized in the latter. Furthermore, particle displacements in these defect grains were strongly correlated in their direction. These results strongly suggest the application of correlation functions in analyzing local stress and local defect grains.

The structural analysis presented in this work is mostly based on a layerwise, 2D analysis. Nevertheless, for an epitaxially grown crystal, defects and dislocations caused by a template-crystal mismatch affect the crystal structure in a 3D way, as also followed from the results presented above. A thorough investigation of stress relaxation in epitaxially grown crystals, especially in the cases where the interlayer density has increased to substantial nonzero values and thus the definition of layers is not strictly applicable anymore, has to include 3D order parameters as well, especially for the larger template-crystal mismatches. By performing a 3D analysis, information about dislocation concentrations, distances between bound dislocation pairs, and correlation lengths can be retrieved.

In a study in which a detailed analysis of defects and dislocations is performed it has to be ensured that the crystals are properly equilibrated during sedimentation. This equilibration crucially depends on the interplay between sedimentation and crystallization and, more specifically, the time scales associated with both processes $[55,56]$. In this study the initial volume fractions were similar to those used in a recent investigation of the crystallization process of sedimenting colloids at a plain hard wall [55]. The time scales associated with the relaxation of structural defects can, however, be much larger than those associated with crystal nucleation and growth [56]. For a more elaborate discussion of equilibration during crystal growth of sedimenting colloids, we refer to Refs. [55,56]. In further research the influence of equilibration on epitaxial colloidal crystal growth has to be investigated as well.

The variation of the $c / a$ ratio by anisotropically stretching the template unit cell starting from either a slightly isotropically shrunken or stretched unit cell compared to the ideal values for epitaxial growth was found to lead to better quality crystals as evidenced by the criteria mentioned above. For both the shrunken and the stretched unit cell, this occurred at a 1.02 stretch of the $c$ axis. For the shrunken unit cell larger deformations finally led to the occurrence of (111)-oriented hexagonally stacked crystals. For the stretched unit cell a non-close-packed superstructure occurred at a 1.50 stretch along the $c$ axis. This structure consisted of regularly stacked crystal planes that followed the stretched hcp (1100) lattice, with the stacking being periodic over six crystal layers. This result even more clearly shows the possibility to create metastable crystal structures using colloidal epitaxy and opens perspectives for the growth of colloidal, hard-sphere quasicrystals.

The stability range of this and possibly other, non-closepacked crystal structures could be broadened when interactions would be made of longer range than purely hardsphere-like. For a bilayer system of particles interacting through a screened repulsive pair potential, Messina and Löwen have recently shown various interesting changes in the phase behavior as a function of screening length [57]. Among these was the notion that crystal structures that are only stable in a small density range in a hard-sphere system become the stable crystal structure over a much larger density window as the range of the interaction is increased. In this respect, the addition of a repulsive component to the particle-particle interactions may pose less strict requirements on the template topography. We have recently demonstrated that for a system of long-range repulsive colloids the template need not be a 2D representation of a crystal plane: a simple 1D pattern was found to already induce the growth of 
crystal structures that would be metastable without the surface pattern [24]. Epitaxial growth on a one-dimensional surface pattern still has to be investigated for a hard-sphere system, but this may already be an indication that bulk metastable structures can be stabilized over a much broader range of the respective parameters in a soft-sphere system than in a hard-sphere system as was shown by Messina and Löwen to be the case for a confined bilayer system.

\section{ACKNOWLEDGMENTS}

We would like to thank Marjolein Dijkstra for a critical reading of the manuscript. This work was part of the research program of the "Stichting voor Fundamenteel Onderzoek der Materie (FOM)," which was financially supported by the "Nederlandse Organisatie voor Wetenschappelijk Onderzoek (NWO).
[1] I. V. Markov, Crystal Growth for Beginners. Fundamentals of Nucleation, Crystal Growth and Epitaxy (World Scientific, Singapore, 1995).

[2] V. J. Anderson and H. N. W. Lekkerkerker, Nature (London) 416, 811 (2002).

[3] P. N. Pusey et al., Phys. Rev. Lett. 63, 2753 (1989).

[4] J. A. Weiss et al., J. Chem. Phys. 103, 1180 (1995).

[5] T. Palberg, Curr. Opin. Colloid Interface Sci. 2, 607 (1997).

[6] U. Gasser et al., Science 292, 258 (2001).

[7] D. H. Van Winkle and C. A. Murray, J. Chem. Phys. 89, 3885 (1988).

[8] D. G. Grier and C. A. Murray, J. Chem. Phys. 100, 9088 (1994).

[9] Y. Tang et al., J. Phys. A 20, L189 (1987).

[10] A. van Blaaderen et al., Langmuir 8, 1514 (1992).

[11] A. van Blaaderen and P. Wiltzius, Science 270, 1177 (1995).

[12] E. R. Weeks et al., Science 287, 627 (2000).

[13] W. K. Kegel and A. van Blaaderen, Science 287, 290 (2000).

[14] A. van Blaaderen, R. Ruel, and P. Wiltzius, Nature (London) 385, 321 (1997).

[15] A. van Blaaderen and P. Wiltzius, Adv. Mater. (Weinheim, Ger.) 9, 833 (1997).

[16] J. P. Hoogenboom et al., Phys. Rev. Lett. 90, 138301 (2003).

[17] Y. N. Xia and G. M. Whitesides, Angew. Chem., Int. Ed. 37, 551 (1998)

[18] J. P. Hoogenboom et al., Appl. Phys. Lett. 80, 4828 (2002).

[19] N. D. Denkov et al., Nature (London) 361, 26 (1993).

[20] P. Jiang et al., Chem. Mater. 11, 2132 (1999).

[21] Y. A. Vlasov et al., Nature (London) 414, 289 (2001).

[22] S. H. Park and Y. N. Xia, Langmuir 15, 266 (1999).

[23] M. Holgado et al., Langmuir 15, 4701 (1999).

[24] J. P. Hoogenboom et al., Phys. Rev. Lett. 89, 256104 (2002).

[25] K.-H. Lin et al., Phys. Rev. Lett. 85, 1770 (2000).

[26] Y. Lu, Y. D. Yin, and Y. N. Xia, Adv. Mater. (Weinheim, Ger.) 13, 34 (2001).

[27] K. P. Velikov et al., Science 296, 106 (2002).

[28] B. J. Alder and T. E. Wainwright, J. Chem. Phys. 27, 1208 (1957)

[29] W. W. Wood and J. D. Jacobson, J. Chem. Phys. 27, 1207 (1957).

[30] S. C. Mau and D. A. Huse, Phys. Rev. E 59, 4396 (1999).

[31] S. Pronk and D. Frenkel, J. Chem. Phys. 110, 4589 (1999).
[32] J. X. Zhu et al., Nature (London) 387, 883 (1997).

[33] N. A. M. Verhaegh et al., J. Chem. Phys. 102, 1416 (1995).

[34] M. S. Elliot, B. T. F. Bristol, and W. C. K. Poon, Physica A 235, 216 (1997).

[35] Z. D. Cheng, W. B. Russell, and P. M. Chaikin, Nature (London) 401, 893 (1999).

[36] C. Dux and H. Versmold, Phys. Rev. Lett. 78, 1811 (1997).

[37] W. K. Kegel and J. K. G. Dhont, J. Chem. Phys. 112, 3431 (2000).

[38] M. Heni and H. Löwen, Phys. Rev. Lett. 85, 3668 (2000).

[39] M. Heni and H. Löwen, J. Phys.: Condens. Matter 13, 4675 (2001).

[40] S. Pronk and D. Frenkel, Phys. Rev. Lett. 90, 255501 (2003).

[41] N. Metoki, W. Donner, and H. Zabel, Phys. Rev. B 49, 17351 (1994).

[42] W. Donner et al., Phys. Rev. B 48, 14745 (1993).

[43] H. Wormeester, E. Huger, and E. Bauer, Phys. Rev. Lett. 77, 1540 (1996).

[44] Y. A. Vlasov et al., Phys. Rev. E 61, 5784 (2000).

[45] V. Yannopapas, N. Stefanou, and A. Modinos, Phys. Rev. Lett. 86, 4811 (2001).

[46] A. van Blaaderen and A. Vrij, Langmuir 8, 2921 (1992).

[47] H. Giesche, J. Eur. Ceram. Soc. 14, 205 (1994).

[48] J. S. van Duijneveldt, J. K. G. Dhont, and H. N. W. Lekkerkerker, J. Chem. Phys. 99, 6941 (1993).

[49] J. C. Crocker and D. G. Grier, J. Colloid Interface Sci. 179, 298 (1996).

[50] C. A. Murray, in Bond-Orientational Order in Condensed Matter Systems, edited by K. J. Strandburg (Springer-Verlag, New York, 1992), p. 137.

[51] M. Schmidt and H. Löwen, Phys. Rev. E 55, 7228 (1997).

[52] S. Neser et al., Phys. Rev. Lett. 79, 2348 (1997).

[53] R. Zangi and S. A. Rice, Phys. Rev. E 61, 660 (2000).

[54] H. Wormeester, E. Huger, and E. Bauer, Phys. Rev. B 57, 10120 (1998).

[55] J. P. Hoogenboom, P. Vergeer, and A. van Blaaderen, J. Chem. Phys. 119, 3371 (2003).

[56] J. P. Hoogenboom, D. Derks, P. Vergeer, and A. van Blaaderen, J. Chem. Phys. 117, 11320 (2002).

[57] R. Messina and H. Löwen, Phys. Rev. Lett. 91, 146101 (2003). 